\title{
ZERO DIELECTRIC CONSTANT LIMIT TO THE NON-ISENTROPIC COMPRESSIBLE EULER-MAXWELL SYSTEM
}

\author{
SONG JIANG AND FUCAI LI*
}

\begin{abstract}
In this paper we investigate the zero dielectric constant limit to the non-isentropic compressible Euler-Maxwell system. We justify this singular limit rigorously in the framework of smooth solutions and obtain the non-isentropic compressible magnetohydrodynamic equations as the dielectric constant tends to zero.
\end{abstract}

\section{Introduction and Main Results}

The electromagnetic dynamics is governing by a coupled PDE system describing the behavior of an electrically conducting fluid and the electromagnetic fields. In the absence of viscosity, Hall effect, and heat conductivity, the system of electromagnetic dynamics can be written as ( $[5,8])$

$$
\begin{aligned}
& \partial_{t} \rho+\operatorname{div}(\rho \mathbf{u})=0, \\
& \rho\left(\partial_{t} \mathbf{u}+\mathbf{u} \cdot \nabla \mathbf{u}\right)+\nabla p=\rho_{\mathrm{e}} \mathbf{E}+\mu_{0} \mathbf{J} \times \mathbf{H}, \\
& \rho \theta\left(\partial_{t} S+\mathbf{u} \cdot \nabla S\right)=\left(\mathbf{J}-\rho_{\mathrm{e}} \mathbf{u}\right) \cdot\left(\mathbf{E}+\mu_{0} \mathbf{u} \times \mathbf{H}\right), \\
& \epsilon \partial_{t} \mathbf{E}-\operatorname{curl} \mathbf{H}+\mathbf{J}=0 \\
& \partial_{t} \mathbf{H}+\frac{1}{\mu_{0}} \operatorname{curl} \mathbf{E}=0 \\
& \partial_{t}\left(\rho_{\mathrm{e}}\right)+\operatorname{div} \mathbf{J}=0 \\
& \epsilon \operatorname{div} \mathbf{E}=\rho_{\mathrm{e}}, \quad \operatorname{div} \mathbf{H}=0 .
\end{aligned}
$$

Here the unknowns $\rho, \mathbf{u}=\left(u_{1}, u_{2}, u_{3}\right) \in \mathbb{R}^{3}, S, \mathbf{E}=\left(E_{1}, E_{2}, E_{3}\right) \in \mathbb{R}^{3}, \mathbf{H}=\left(H_{1}\right.$, $\left.H_{2}, H_{3}\right) \in \mathbb{R}^{3}$, and $\rho_{\mathrm{e}}$ denote the density, velocity, entropy, electric field, magnetic field, and electric charge density, respectively. The current density $\mathbf{J}$ is expressed by Ohm's law, i.e.,

$$
\mathbf{J}-\rho_{\mathrm{e}} \mathbf{u}=\sigma\left(\mathbf{E}+\mu_{0} \mathbf{u} \times \mathbf{H}\right) .
$$

The pressure $p$ and the entropy $S$ satisfy the Gibbs relation

$$
\theta \mathrm{d} S=\mathrm{d} e+p \mathrm{~d}\left(\frac{1}{\rho}\right)
$$

where $\theta$ and $e$ denote the temperature and the internal energy of the fluid.

Date: January 17, 2021.

2000 Mathematics Subject Classification. 76W05, 35Q60, 35B25.

Key words and phrases. non-isentropic compressible Euler-Maxwell system, non-isentropic compressible magnetohydrodynamic equations, zero dielectric constant limit, nonlinear energy method.

* Corresponding author. 
To the authors' best knownledge, the only mathematical result on the system (1.1) - (1.7) was obtained by Kawashima [14 who established the global existence of smooth solutions in whole space $\mathbb{R}^{2}$ when the initial data are a small perturbation of some given constant state. On the other hand, as it was pointed out in [8], the assumption that the electric charge density $\rho_{\mathrm{e}} \simeq 0$ is physically very reasonable for the study of plasmas. In this situation, we can eliminate the terms involving $\rho_{\mathrm{e}}$ in (1.1)-1.7) and then obtain the following non-isentropic compressible EulerMaxwell system:

$$
\begin{aligned}
& \partial_{t} \rho+\operatorname{div}(\rho \mathbf{u})=0, \\
& \rho\left(\partial_{t} \mathbf{u}+\mathbf{u} \cdot \nabla \mathbf{u}\right)+\nabla P=\mu_{0} \mathbf{J} \times \mathbf{H}, \\
& \rho \theta\left(\partial_{t} S+\mathbf{u} \cdot \nabla S\right)=\mathbf{J} \cdot\left(\mathbf{E}+\mu_{0} \mathbf{u} \times \mathbf{H}\right), \\
& \epsilon \partial_{t} \mathbf{E}-\operatorname{curl} \mathbf{H}+\mathbf{J}=0, \\
& \partial_{t} \mathbf{H}+\frac{1}{\mu_{0}} \operatorname{curl} \mathbf{E}=0, \quad \operatorname{div} \mathbf{H}=0
\end{aligned}
$$

with

$$
\mathbf{J}=\sigma\left(\mathbf{E}+\mu_{0} \mathbf{u} \times \mathbf{H}\right) .
$$

Formally, if we take the dielectric constant $\epsilon=0$ in (1.13), i.e., the displacement current is negligible, then we obtain $\mathbf{J}=\operatorname{curl} \mathbf{H}$. Thanks to (1.15), we can eliminate the electric field $\mathbf{E}$ in (1.11), (1.12) and (1.14), and finally obtain that

$$
\begin{aligned}
& \partial_{t} \rho+\operatorname{div}(\rho \mathbf{u})=0, \\
& \rho\left(\partial_{t} \mathbf{u}+\mathbf{u} \cdot \nabla \mathbf{u}\right)+\nabla P=\mu_{0} \operatorname{curl} \mathbf{H} \times \mathbf{H}, \\
& \rho \theta\left(\partial_{t} S+\mathbf{u} \cdot \nabla S\right)=\frac{1}{\sigma}|\operatorname{curl} \mathbf{H}|^{2}, \\
& \partial_{t} \mathbf{H}-\operatorname{curl}(\mathbf{u} \times \mathbf{H})=-\frac{1}{\sigma \mu_{0}} \operatorname{curl}(\operatorname{curl} \mathbf{H}), \quad \operatorname{div} \mathbf{H}=0 .
\end{aligned}
$$

The equations (1.16) - (1.19) is called non-isentropic compressible magnetohydrodynamic equations with infinite Reynolds number which is used in describing some local processes in the cosmic system [6].

The above formal derivation is usually referred as magnetohydrodynamic approximation [5, 8. In [15, 16, Kawashima and Shizuta justified this limit process rigorously to the complete magnetohydrodynamic fluid system in $\mathbb{R}^{2}$ for local and global small smooth solutions (small perturbations of some give constant state), respectively. In [12, we studied the magnetohydrodynamic approximation for the isentropic electromagnetic fluid system in three-dimensional period domain and obtained the isentropic compressible magnetohydrodynamic equations with explicit convergence rates. Recently, we extended the results in [12] to the complete magnetohydrodynamic fluid system and obtained the full compressible magnetohydrodynamic equations [13. We remark that the viscosities (including the shear and buck viscosities and heat conductivity coefficient) play a crucial role in the proof process of [13] and the inviscid case is left as an open problem there.

The purpose of this paper is to solve this problem and give a rigorous derivation of the compressible magnetohydrodynamic equations (1.16) (1.19) from the non-isentropic compressible Euler-Maxwell system (1.10)-11.15) as the dielectric constant $\epsilon$ tends to zero. As in [13, we consider the system (1.10)-1.15) in a periodic domain of $\mathbb{R}^{3}$, i.e., the torus $\mathbb{T}^{3}=(\mathbb{R} /(2 \pi \mathbb{Z}))^{3}$. 
Below we take the harmless physical constants $\sigma$ and $\mu_{0}$ to be one for simplicity of presentation. For the system (1.10)-(1.15), it is more convenient to using the pressure $p$ instead of the density $\rho$ as an unknown. Thus we reconsider the equations of state as functions of $S$ and $p$, i.e., $\rho=r(S, p)$ and $\theta=\Theta(S, p)$ for some positive smooth functions $r$ and $\Theta$ defined for all $S$ and $p>0$, and satisfying $\frac{\partial r(S, p)}{\partial p}>0$. Moreover, in order to emphasize the unknowns depending on the small parameter $\epsilon$, we add the superscripts $\epsilon$ to the unknowns $(p, \mathbf{u}, S, \mathbf{E}, \mathbf{H})$ and rewrite the EulerMaxwell system (1.10)-1.15) as

$$
\begin{aligned}
& a\left(S^{\epsilon}, p^{\epsilon}\right)\left(\partial_{t} p^{\epsilon}+\mathbf{u}^{\epsilon} \cdot \nabla p^{\epsilon}\right)+\operatorname{div} \mathbf{u}^{\epsilon}=0, \\
& r\left(S^{\epsilon}, p^{\epsilon}\right)\left(\partial_{t} \mathbf{u}^{\epsilon}+\mathbf{u}^{\epsilon} \cdot \nabla \mathbf{u}^{\epsilon}\right)+\nabla p^{\epsilon}=\left(\mathbf{E}^{\epsilon}+\mathbf{u}^{\epsilon} \times \mathbf{H}^{\epsilon}\right) \times \mathbf{H}^{\epsilon}, \\
& b\left(S^{\epsilon}, p^{\epsilon}\right)\left(\partial_{t} S^{\epsilon}+\mathbf{u}^{\epsilon} \cdot \nabla S^{\epsilon}\right)=\left|\mathbf{E}^{\epsilon}+\mathbf{u}^{\epsilon} \times \mathbf{H}^{\epsilon}\right|^{2}, \\
& \epsilon \partial_{t} \mathbf{E}^{\epsilon}-\operatorname{curl} \mathbf{H}^{\epsilon}+\left(\mathbf{E}^{\epsilon}+\mathbf{u}^{\epsilon} \times \mathbf{H}^{\epsilon}\right)=0 \\
& \partial_{t} \mathbf{H}^{\epsilon}+\operatorname{curl} \mathbf{E}^{\epsilon}=0, \quad \operatorname{div} \mathbf{H}^{\epsilon}=0 .
\end{aligned}
$$

where $a\left(S^{\epsilon}, p^{\epsilon}\right)$ and $b\left(S^{\epsilon}, p^{\epsilon}\right)$ are defined as

$$
a\left(S^{\epsilon}, p^{\epsilon}\right)=\frac{1}{r\left(S^{\epsilon}, p^{\epsilon}\right)} \frac{\partial r\left(S^{\epsilon}, p^{\epsilon}\right)}{\partial p^{\epsilon}}, \quad b\left(S^{\epsilon}, p^{\epsilon}\right)=r\left(S^{\epsilon}, p^{\epsilon}\right) \Theta\left(S^{\epsilon}, p^{\epsilon}\right) .
$$

The system (1.20)-1.24) are supplemented with initial data

$$
\left.\left(p^{\epsilon}, \mathbf{u}^{\epsilon}, S^{\epsilon}, \mathbf{E}^{\epsilon}, \mathbf{H}^{\epsilon}\right)\right|_{t=0}=\left(p_{0}^{\epsilon}(x), \mathbf{u}_{0}^{\epsilon}(x), S_{0}^{\epsilon}(x), \mathbf{E}_{0}^{\epsilon}(x), \mathbf{H}_{0}^{\epsilon}(x)\right), \quad x \in \mathbb{T}^{3} .
$$

We also rewrite the target equations (1.16)-(1.19) (recall that $\mu_{0} \equiv \sigma \equiv 1$ ) as

$$
\begin{aligned}
& a\left(S^{0}, p^{0}\right)\left(\partial_{t} p^{0}+\mathbf{u}^{0} \cdot \nabla p^{0}\right)+\operatorname{div} \mathbf{u}^{0}=0, \\
& r\left(S^{0}, p^{0}\right)\left(\partial_{t} \mathbf{u}^{0}+\mathbf{u}^{0} \cdot \nabla \mathbf{u}^{0}\right)+\nabla p^{0}=\operatorname{curl} \mathbf{H}^{0} \times \mathbf{H}^{0}, \\
& b\left(S^{0}, p^{0}\right)\left(\partial_{t} S^{0}+\mathbf{u}^{0} \cdot \nabla S^{0}\right)=\left|\operatorname{curl} \mathbf{H}^{0}\right|^{2}, \\
& \partial_{t} \mathbf{H}^{0}-\operatorname{curl}\left(\mathbf{u}^{0} \times \mathbf{H}^{0}\right)=-\operatorname{curlcurl} \mathbf{H}^{0}, \quad \operatorname{div} \mathbf{H}^{0}=0 .
\end{aligned}
$$

where $a\left(S^{0}, p^{0}\right)$ and $b\left(S^{0}, p^{0}\right)$ are defined through 1.25) with $\left(S^{\epsilon}, p^{\epsilon}\right)$ replaced by $\left(S^{0}, p^{0}\right)$. The system (1.27)-(1.30) are equipped with initial data

$$
\left.\left(p^{0}, \mathbf{u}^{0}, S^{0}, \mathbf{H}^{0}\right)\right|_{t=0}=\left(p_{0}^{0}(x), \mathbf{u}_{0}^{0}(x), S_{0}^{0}(x), \mathbf{H}_{0}^{0}(x)\right), \quad x \in \mathbb{T}^{3} .
$$

We remark that although the electric field $\mathbf{E}^{0}$ does not appear in the system (1.27)-1.30), it can be induced according to the relation

$$
\mathbf{E}^{0}=\operatorname{curl} \mathbf{H}^{0}-\mathbf{u}^{0} \times \mathbf{H}^{0}
$$

by the moving conductive flow in the magnetic field.

Before stating our main results, we recall the local existence of smooth solutions to the problem (1.27)-1.31). Since the system (1.27)-(1.30) can be written as a symmetric hyperbolic-parabolic system, the results in 26] imply that

Proposition 1.1. Let $s>7 / 2$ be an integer and assume that the initial data $\left(p_{0}^{0}, \mathbf{u}_{0}^{0}, S_{0}^{0}, \mathbf{H}_{0}^{0}\right)$ satisfy

$$
\begin{gathered}
p_{0}^{0}, \mathbf{u}_{0}^{0}, S_{0}^{0}, \mathbf{H}_{0}^{0} \in H^{s+1}\left(\mathbb{T}^{3}\right), \quad \operatorname{div} \mathbf{H}_{0}^{0}=0, \\
0<\bar{p}=\inf _{x \in \mathbb{T}^{3}} p_{0}^{0}(x) \leqslant p_{0}^{0}(x) \leqslant \overline{\bar{p}}=\sup _{x \in \mathbb{T}^{3}} p_{0}^{0}(x)<+\infty, \\
0<\bar{S}=\inf _{x \in \mathbb{T}^{3}} S_{0}^{0}(x) \leqslant S_{0}^{0}(x) \leqslant \overline{\bar{S}}=\sup _{x \in \mathbb{T}^{3}} S_{0}^{0}(x)<+\infty
\end{gathered}
$$


for some positive constants $\bar{p}, \overline{\bar{p}}, \bar{S}$, and $\overline{\bar{S}}$. Then there exist positive constants $T_{*}$ (the maximal time interval, $0<T_{*} \leqslant+\infty$ ) and $\hat{p}, \tilde{p}, \hat{S}, \tilde{S}$, such that the problem (1.27) -1.31) has a unique classical solution $\left(p^{0}, \mathbf{u}^{0}, S^{0}, \mathbf{H}^{0}\right)$ satisfying $\operatorname{div} \mathbf{H}^{0}=0$ and

$$
\begin{gathered}
p^{0}, \mathbf{u}^{0}, S^{0} \in C^{l}\left(\left[0, T_{*}\right), H^{s+1-l}\left(\mathbb{T}^{3}\right)\right), \mathbf{H}^{0} \in C^{l}\left(\left[0, T_{*}\right), H^{s+1-2 l}\left(\mathbb{T}^{3}\right)\right), \quad l=0,1 ; \\
0<\hat{p}=\inf _{(x, t) \in \mathbb{T}^{3} \times\left[0, T_{*}\right)} p^{0}(x, t) \leqslant p^{0}(x, t) \leqslant \tilde{p}=\sup _{(x, t) \in \mathbb{T}^{3} \times\left[0, T_{*}\right)} p^{0}(x, t)<+\infty, \\
0<\hat{S}=\inf _{(x, t) \in \mathbb{T}^{3} \times\left[0, T_{*}\right)} S^{0}(x, t) \leqslant S^{0}(x, t) \leqslant \tilde{S}=\sup _{(x, t) \in \mathbb{T}^{3} \times\left[0, T_{*}\right)} S^{0}(x, t)<+\infty .
\end{gathered}
$$

The main result of this paper can be stated as follows.

Theorem 1.2. Let $s>7 / 2$ be an integer and $\left(p^{0}, \mathbf{u}^{0}, S^{0}, \mathbf{H}^{0}\right)$ the unique classical solution to the problem (1.27)-(1.31) given in Proposition 1.1. Suppose that the initial data $\left(p_{0}^{\epsilon}, \mathbf{u}_{0}^{\epsilon}, S_{0}^{\epsilon}, \mathbf{E}_{0}^{\epsilon}, \mathbf{H}_{0}^{\epsilon}\right)$ satisfy

$$
p_{0}^{\epsilon}, \mathbf{u}_{0}^{\epsilon}, S_{0}^{\epsilon}, \mathbf{E}_{0}^{\epsilon}, \mathbf{H}_{0}^{\epsilon} \in H^{s}\left(\mathbb{T}^{3}\right), \inf _{x \in \mathbb{T}^{3}} p_{0}^{\epsilon}(x)>0, \inf _{x \in \mathbb{T}^{3}} S_{0}^{\epsilon}(x)>0, \operatorname{div} \mathbf{H}_{0}^{\epsilon}=0,
$$

and

$$
\begin{aligned}
\|\left(p_{0}^{\epsilon}-p_{0}^{0}, \mathbf{u}_{0}^{\epsilon}-\mathbf{u}_{0}^{0},\right. & \left.S_{0}^{\epsilon}-S_{0}^{0}, \mathbf{H}_{0}^{\epsilon}-\mathbf{H}_{0}^{0}\right) \|_{H^{s}\left(\mathbb{T}^{3}\right)} \\
& +\sqrt{\epsilon}\left\|\mathbf{E}_{0}^{\epsilon}-\left(\operatorname{curl} \mathbf{H}_{0}^{0}-\mathbf{u}_{0}^{0} \times \mathbf{H}_{0}^{0}\right)\right\|_{H^{s}\left(\mathbb{T}^{3}\right)} \leqslant L_{0} \epsilon
\end{aligned}
$$

for some constant $L_{0}>0$. Then, for any $T_{0} \in\left(0, T_{*}\right)$, there exist a constant $L>0$, and a sufficient small constant $\epsilon_{0}>0$ such that, for any $\epsilon \in\left(0, \epsilon_{0}\right]$, the problem (1.20) -(1.26) has a unique smooth solution $\left(p^{\epsilon}, \mathbf{u}^{\epsilon}, S^{\epsilon}, \mathbf{E}^{\epsilon}, \mathbf{H}^{\epsilon}\right)$ on $\left[0, T_{0}\right]$ enjoying

$$
\begin{aligned}
& \left\|\left(p^{\epsilon}-p^{0}, \mathbf{u}^{\epsilon}-\mathbf{u}^{0}, S^{\epsilon}-S^{0}, \mathbf{H}^{\epsilon}-\mathbf{H}^{0}\right)(t)\right\|_{H^{s}\left(\mathbb{T}^{3}\right)} \\
& +\sqrt{\epsilon}\left\|\left\{\mathbf{E}^{\epsilon}-\left(\operatorname{curl} \mathbf{H}^{0}-\mathbf{u}^{0} \times \mathbf{H}^{0}\right)\right\}(t)\right\|_{H^{s}\left(\mathbb{T}^{3}\right)} \leqslant L \epsilon, \quad t \in\left[0, T_{0}\right] .
\end{aligned}
$$

We shall prove Theorem 1.2 by adapting the elaborate nonlinear energy method inspired by [12,13. The key point of the proof is to derive the error system (see (2.1) - (2.5) below) and obtain the uniform estimates in a fixed time interval independent of $\epsilon$. As mentioned before, the zero dielectric constant limit to the complete magnetohydrodynamic fluid system were studied in [13] where the viscosity and heat conductivity terms in the complete electromagnetic fluid system play a crucial role in the derivation of the uniform estimates. In our case, all diffusion terms disappear and we shall make full use of the special structural of the system (1.20)(1.23) to obtain the desired uniformly estimates. A direct but crucial observation is that there is a damping term $\mathbf{E}^{\epsilon}-\mathbf{E}^{0}$ in the electric field equations which control the terms involving $\mathbf{E}^{\epsilon}-\mathbf{E}^{0}$ in the momentum equations, entropy equation, and electric filed equations. In order to obtain the desired higher order estimates to the error system, we shall also modify some ideas developed in [10,20 which is quite different to the isentropic case [12] and the viscous non-isentropic case [13.

Remark 1.1. The inequality (1.34) implies that the sequences $\left(p^{\epsilon}, \mathbf{u}^{\epsilon}, S^{\epsilon}, \mathbf{H}^{\epsilon}\right)$ converge strongly to $\left(p^{0}, \mathbf{u}^{0}, S^{0}, \mathbf{H}^{0}\right)$ in $L^{\infty}\left(0, T ; H^{s}\left(\mathbb{T}^{3}\right)\right)$ and $\mathbf{E}^{\epsilon}$ converges strongly to $\mathbf{E}^{0}$ in $L^{\infty}\left(0, T ; H^{s}\left(\mathbb{T}^{3}\right)\right)$ but with different convergence rates, where $\mathbf{E}^{0}$ is defined by (1.32). 
Remark 1.2. For the local existence of solutions $\left(p^{0}, \mathbf{u}^{0}, S^{0}, \mathbf{H}^{0}\right)$ to the problem (1.27) -(1.31), the assumption on the regularity of initial data $\left(p_{0}^{0}, \mathbf{u}_{0}^{0}, \theta_{0}^{0}, \mathbf{H}_{0}^{0}\right)$ belongs to $H^{s}\left(\mathbb{T}^{3}\right), s>7 / 2$, is enough. Here we have added more regularity assumption in Proposition 1.1 to obtain more regular solutions which are needed in the proof of Theorem 1.2. The higher regularity assumption on the target equations can provide a simpler arguments in this paper. To investigate the singular limit, another way is to obtain higher order uniform estimates directly, see, for example, 20. on zero Mach number limit to non-isentropic Euler equations.

Remark 1.3. In this paper we just consider the periodic domain case, it is more interesting to study the same problem in a spatial domain with boundary which will be our future study. We remark that in this case the boundary must be analyzed very carefully, the interested reader can refer [1,4,23,25], and among others on the zero Mach number limit of the compressible Euler equations, and 22] on singular limits of zero Alfvén number for the equations of magneto-fluid dynamics. In [2, 3], some new pioneering ideas are introduced, which can be applied to the convergence study of the singular limit in the data space, see [3,4,22 for the details.

Remark 1.4. We point out that the zero dielectric constant limit is a singular limit and similar to the zero Mach number limit in some sense, see [1,4,9,11,17,20,23,25] and the references cited therein.

Remark 1.5. It is obvious that if we let $\sigma \rightarrow \infty$ in (1.16)-1.19), we will obtain formally the well-known ideal non-isentropic magnetohydrodynamic equations. It is interesting to establish this limit rigorously.

Before ending this introduction, we give some notations and recall some basic facts which will be frequently used throughout this paper.

(1) We denote by $\langle\cdot, \cdot\rangle$ the standard inner product in $L^{2}\left(\mathbb{T}^{3}\right)$ with $\langle f, f\rangle=\|f\|^{2}$, by $H^{k}$ the standard Sobolev space $W^{k, 2}$ with $\|\cdot\|_{k}$ being the corresponding norm $\left(\|\cdot\|_{0} \equiv\|\cdot\|\right)$. The notation $\left\|\left(A_{1}, A_{2}, \ldots, A_{k}\right)\right\|$ means the summation of $\left\|A_{i}\right\|, i=$ $1, \ldots, k$, and it also applies to other norms. For the multi-index $\alpha=\left(\alpha_{1}, \alpha_{2}, \alpha_{3}\right)$, we denote $\partial_{x}^{\alpha}=\partial_{x_{1}}^{\alpha_{1}} \partial_{x_{2}}^{\alpha_{2}} \partial_{x_{3}}^{\alpha_{3}}$ and $|\alpha|=\left|\alpha_{1}\right|+\left|\alpha_{2}\right|+\left|\alpha_{3}\right|$. For the integer $l$, the symbol $D_{x}^{l}$ denotes the summation of all terms $\partial_{x}^{\alpha}$ with the multi-index $\alpha$ satisfying $|\alpha|=l$. We use $C_{i}, \delta_{i}, K_{i}$, and $K$ to denote the constants which are independent of $\epsilon$ and may change from line to line. We also omit the spatial domain $\mathbb{T}^{3}$ in integrals for convenience.

(2) We shall frequently use the following Moser-type calculus inequalities (see [17):

(i) For $f, g \in H^{s}\left(\mathbb{T}^{3}\right) \cap L^{\infty}\left(\mathbb{T}^{3}\right)$ and $|\alpha| \leqslant s, s>3 / 2$, it holds that

$$
\left\|\partial_{x}^{\alpha}(f g)\right\| \leqslant C_{s}\left(\|f\|_{L^{\infty}}\left\|D_{x}^{s} g\right\|+\|g\|_{L^{\infty}}\left\|D_{x}^{s} f\right\|\right) .
$$

(ii) For $f \in H^{s}\left(\mathbb{T}^{3}\right), D_{x}^{1} f \in L^{\infty}\left(\mathbb{T}^{3}\right), g \in H^{s-1}\left(\mathbb{T}^{3}\right) \cap L^{\infty}\left(\mathbb{T}^{3}\right)$ and $|\alpha| \leqslant s$, $s>5 / 2$, it holds that

$$
\left\|\partial_{x}^{\alpha}(f g)-f \partial_{x}^{\alpha} g\right\| \leqslant C_{s}\left(\left\|D_{x}^{1} f\right\|_{L^{\infty}}\left\|D_{x}^{s-1} g\right\|+\|g\|_{L^{\infty}}\left\|D_{x}^{s} f\right\|\right) .
$$

(3) Let $s>3 / 2, f \in C^{s}\left(\mathbb{T}^{3}\right)$, and $u \in H^{s}\left(\mathbb{T}^{3}\right)$, then for each multi-index $\alpha$, $1 \leqslant|\alpha| \leqslant s$, we have $([17,18])$ :

$$
\left\|\partial_{x}^{\alpha}(f(u))\right\| \leqslant C\left(1+\|u\|_{L^{\infty}}^{|\alpha|-1}\right)\|u\|_{|\alpha|} ;
$$


moreover, if $f(0)=0$, then $([7])$

$$
\left\|\partial_{x}^{\alpha}(f(u))\right\| \leqslant C\left(\|u\|_{s}\right)\|u\|_{s} .
$$

This paper is organized as follows. In Section 2, we utilize the primitive system (1.20)-(1.24) and the target system (1.27)-1.30) to derive an error system and state the local existence of solutions to the error system. In Section 3 we give $a$ priori energy estimates to the error system and present the proof of Theorem 1.2

\section{Derivation of AN ERROR SYStem AND LOCAL EXISTENCE}

In this section we first derive an error system from the original system (1.20)(1.24) and the target equations (1.27)-(1.30). Then we state the local existence of smooth solutions to this error system.

Setting $P^{\epsilon}=p^{\epsilon}-p^{0}, \mathbf{U}^{\epsilon}=\mathbf{u}^{\epsilon}-\mathbf{u}^{0}, \Phi^{\epsilon}=S^{\epsilon}-S^{0}, \mathbf{F}^{\epsilon}=\mathbf{E}^{\epsilon}-\mathbf{E}^{0}, \mathbf{G}^{\epsilon}=\mathbf{H}^{\epsilon}-\mathbf{H}^{0}$ and utilizing the system (1.20)-(1.24) and the system (1.27)-(1.30) with (1.32), we obtain that

$$
\begin{aligned}
& a\left(\Phi^{\epsilon}+S^{0}, P^{\epsilon}+p^{0}\right)\left\{\partial_{t} P^{\epsilon}+\left(\mathbf{U}^{\epsilon}+\mathbf{u}^{0}\right) \cdot \nabla P^{\epsilon}\right\}+\operatorname{div} \mathbf{U}^{\epsilon}=f_{1}^{\epsilon}, \\
& r\left(\Phi^{\epsilon}+S^{0}, P^{\epsilon}+p^{0}\right)\left\{\partial_{t} \mathbf{U}^{\epsilon}+\left(\mathbf{U}^{\epsilon}+\mathbf{u}^{0}\right) \cdot \nabla \mathbf{U}^{\epsilon}\right\}+\nabla \Phi^{\epsilon}=\mathbf{f}_{2}^{\epsilon}, \\
& b\left(\Phi^{\epsilon}+S^{0}, P^{\epsilon}+p^{0}\right)\left\{\partial_{t} \Phi^{\epsilon}+\left(\mathbf{U}^{\epsilon}+\mathbf{u}^{0}\right) \cdot \nabla \Phi^{\epsilon}\right\}=\mathbf{f}_{3}^{\epsilon}, \\
& \epsilon \partial_{t} \mathbf{F}^{\epsilon}-\operatorname{curl} \mathbf{G}^{\epsilon}=\mathbf{f}_{4}^{\epsilon}, \\
& \partial_{t} \mathbf{G}^{\epsilon}+\operatorname{curl} \mathbf{F}^{\epsilon}=0, \quad \operatorname{div} \mathbf{G}^{\epsilon}=0,
\end{aligned}
$$

where $f_{1}^{\epsilon}, \mathbf{f}_{2}^{\epsilon}, \mathbf{f}_{3}^{\epsilon}$, and $\mathbf{f}_{4}^{\epsilon}$ are defined as follows:

$$
\begin{aligned}
f_{1}^{\epsilon}= & -\left[a\left(\Phi^{\epsilon}+S^{0}, P^{\epsilon}+p^{0}\right)-a\left(S^{0}, p^{0}\right)\right]\left[\partial_{t} p^{0}+\mathbf{u}^{0} \cdot \nabla p^{0}\right] \\
& -a\left(\Phi^{\epsilon}+S^{0}, P^{\epsilon}+p^{0}\right)\left(\mathbf{U}^{\epsilon} \cdot \nabla p^{0}\right), \\
\mathbf{f}_{2}^{\epsilon}= & -\left[r\left(\Phi^{\epsilon}+S^{0}, P^{\epsilon}+p^{0}\right)-r\left(S^{0}, p^{0}\right)\right]\left[\partial_{t} \mathbf{u}^{0}+\mathbf{u}^{0} \cdot \nabla \mathbf{u}^{0}\right] \\
& -r\left(\Phi^{\epsilon}+S^{0}, P^{\epsilon}+p^{0}\right)\left(\mathbf{U}^{\epsilon} \cdot \nabla \mathbf{u}^{0}\right) \\
& -\operatorname{curl} \mathbf{H}^{0} \times \mathbf{H}^{0}+\left[\mathbf{F}^{\epsilon}+\mathbf{u}^{0} \times \mathbf{G}^{\epsilon}+\mathbf{U}^{\epsilon} \times \mathbf{H}^{0}\right] \times \mathbf{H}^{0} \\
& +\left[\mathbf{F}^{\epsilon}+\mathbf{u}^{0} \times \mathbf{G}^{\epsilon}+\mathbf{U}^{\epsilon} \times \mathbf{H}^{0}\right] \times \mathbf{G}^{\epsilon}+\left(\mathbf{U}^{\epsilon} \times \mathbf{G}^{\epsilon}\right) \times\left(\mathbf{G}^{\epsilon}+\mathbf{H}^{0}\right), \\
\mathbf{f}_{3}^{\epsilon}= & -\left[b\left(\Phi^{\epsilon}+S^{0}, P^{\epsilon}+p^{0}\right)-b\left(S^{0}, p^{0}\right)\right]\left[\partial_{t} S^{0}+\mathbf{u}^{0} \cdot \nabla S^{0}\right] \\
& -b\left(\Phi^{\epsilon}+S^{0}, P^{\epsilon}+p^{0}\right)\left(\mathbf{U}^{\epsilon} \cdot \nabla S^{0}\right) \\
& +\left|\mathbf{F}^{\epsilon}+\mathbf{U}^{\epsilon} \times \mathbf{G}^{\epsilon}\right|^{2}+\left|\mathbf{u}^{0} \times \mathbf{G}^{\epsilon}+\mathbf{U}^{\epsilon} \times \mathbf{H}^{0}\right|^{2} \\
& +2\left(\mathbf{F}^{\epsilon}+\mathbf{U}^{\epsilon} \times \mathbf{G}^{\epsilon}\right) \cdot\left[\operatorname{curl} \mathbf{H}^{0}+\mathbf{u}^{0} \times \mathbf{G}^{\epsilon}+\mathbf{U}^{\epsilon} \times \mathbf{H}^{0}\right] \\
& +2 \operatorname{curl} \mathbf{H}^{0} \cdot\left(\mathbf{u}^{0} \times \mathbf{G}^{\epsilon}+\mathbf{U}^{\epsilon} \times \mathbf{H}^{0}\right), \\
\mathbf{f}_{4}^{\epsilon}= & -\left[\mathbf{F}^{\epsilon}+\mathbf{U}^{\epsilon} \times \mathbf{H}^{0}+\mathbf{u}^{0} \times \mathbf{G}^{\epsilon}\right]-\mathbf{U}^{\epsilon} \times \mathbf{G}^{\epsilon} \\
& -\epsilon \partial_{t} \operatorname{curl} \mathbf{H}^{0}+\epsilon \partial_{t}\left(\mathbf{u}^{0} \times \mathbf{H}^{0}\right) .
\end{aligned}
$$

The system (2.1)-(2.5) are supplemented with initial data

$$
\begin{aligned}
& \left.\left(P^{\epsilon}, \mathbf{U}^{\epsilon}, \Phi^{\epsilon}, \mathbf{F}^{\epsilon}, \mathbf{G}^{\epsilon}\right)\right|_{t=0}=\left(P_{0}^{\epsilon}, \mathbf{U}_{0}^{\epsilon}, \Phi_{0}^{\epsilon}, \mathbf{F}_{0}^{\epsilon}, \mathbf{G}_{0}^{\epsilon}\right) \\
& \quad:=\left(p_{0}^{\epsilon}-p_{0}^{0}, \mathbf{u}_{0}^{\epsilon}-\mathbf{u}_{0}^{0}, S_{0}^{\epsilon}-S_{0}^{0}, \mathbf{E}_{0}^{\epsilon}-\left(\operatorname{curl} \mathbf{H}_{0}^{0}-\mathbf{u}_{0}^{0} \times \mathbf{H}_{0}^{0}\right), \mathbf{H}_{0}^{\epsilon}-\mathbf{H}_{0}^{0}\right) .
\end{aligned}
$$


Denote

$$
\begin{aligned}
& \mathbf{W}^{\epsilon}=\left(\begin{array}{c}
P^{\epsilon} \\
\mathbf{U}^{\epsilon} \\
\Phi^{\epsilon} \\
\mathbf{F}^{\epsilon} \\
\mathbf{G}^{\epsilon}
\end{array}\right), \quad \mathbf{W}_{0}^{\epsilon}=\left(\begin{array}{c}
P_{0}^{\epsilon} \\
\mathbf{U}_{0}^{\epsilon} \\
\Phi_{0}^{\epsilon} \\
\mathbf{F}_{0}^{\epsilon} \\
\mathbf{G}_{0}^{\epsilon}
\end{array}\right), \quad \mathbf{S}^{\epsilon}\left(\mathbf{W}^{\epsilon}\right)=\left(\begin{array}{c}
f_{1}^{\epsilon} \\
\mathbf{f}_{2}^{\epsilon} \\
\mathbf{f}_{3}^{\epsilon} \\
\mathbf{f}_{4}^{\epsilon} \\
\mathbf{0}
\end{array}\right),
\end{aligned}
$$

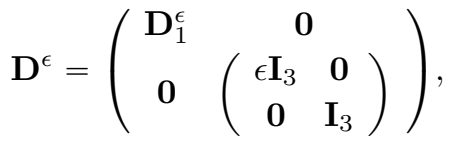

$$
\begin{aligned}
& \mathbf{D}_{1}^{\epsilon}=\left(\begin{array}{ccc}
a\left(\Phi^{\epsilon}+S^{0}, P^{\epsilon}+p^{0}\right) & 0 & 0 \\
0 & r\left(\Phi^{\epsilon}+S^{0}, P^{\epsilon}+p^{0}\right) \mathbf{I}_{3} & \mathbf{0} \\
0 & \mathbf{0} & b\left(\Phi^{\epsilon}+S^{0}, P^{\epsilon}+p^{0}\right)
\end{array}\right),
\end{aligned}
$$

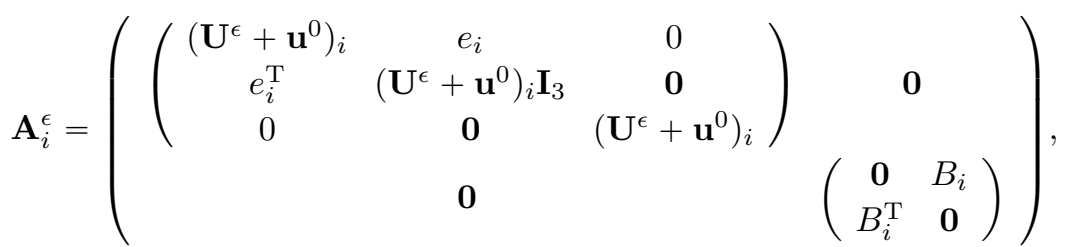

where $\left(e_{1}, e_{2}, e_{3}\right)$ is the canonical basis of $\mathbb{R}^{3}, \mathbf{I}_{d}(d=3,5)$ is the $d \times d$ unit matrix, $y_{i}$ denotes the $i$-th component of $y \in \mathbb{R}^{3}$, and

$$
B_{1}=\left(\begin{array}{ccc}
0 & 0 & 0 \\
0 & 0 & 1 \\
0 & -1 & 0
\end{array}\right), \quad B_{2}=\left(\begin{array}{ccc}
0 & 0 & -1 \\
0 & 0 & 0 \\
1 & 0 & 0
\end{array}\right), \quad B_{3}=\left(\begin{array}{ccc}
0 & 1 & 0 \\
-1 & 0 & 0 \\
0 & 0 & 0
\end{array}\right)
$$

Using these notations we can rewrite the problem (2.1)-(2.6) as

$$
\left\{\begin{array}{l}
\mathbf{D}^{\epsilon} \partial_{t} \mathbf{W}^{\epsilon}+\sum_{i=1}^{3} \mathbf{A}_{i}^{\epsilon} \mathbf{W}_{x_{i}}^{\epsilon}=\mathbf{S}^{\epsilon}\left(\mathbf{W}^{\epsilon}\right), \\
\left.\mathbf{W}^{\epsilon}\right|_{t=0}=\mathbf{W}_{0}^{\epsilon}
\end{array}\right.
$$

Obviously, the system in (2.7) is a quasilinear symmetric hyperbolic one. Thus, we can apply the result of Majda [19] to obtain the following local existence of smooth solutions to the problem (2.7).

Proposition 2.1. Let $s>7 / 2$ be an integer and $\left(p_{0}^{0}, \mathbf{u}_{0}^{0}, S_{0}^{0}, \mathbf{H}_{0}^{0}\right)$ satisfy the conditions in Proposition 1.1. Assume that the initial data $\left(P_{0}^{\epsilon}, \mathbf{U}_{0}^{\epsilon}, \Phi_{0}^{\epsilon}, \mathbf{F}_{0}^{\epsilon}, \mathbf{G}_{0}^{\epsilon}\right)$ satisfy

$$
\begin{gathered}
P_{0}^{\epsilon}, \mathbf{U}_{0}^{\epsilon}, \Phi_{0}^{\epsilon}, \mathbf{F}_{0}^{\epsilon}, \mathbf{G}_{0}^{\epsilon} \in H^{s}\left(\mathbb{T}^{3}\right), \quad \operatorname{div} \mathbf{G}_{0}^{\epsilon}=0, \\
\inf _{x \in \mathbb{T}^{3}} P_{0}^{\epsilon}(x)>0, \quad \inf _{x \in \mathbb{T}^{3}} \Phi_{0}^{\epsilon}(x)>0, \quad\left\|\Phi_{0}^{\epsilon}\right\|_{s} \leqslant \delta, \quad\left\|P_{0}^{\epsilon}\right\|_{s} \leqslant \delta
\end{gathered}
$$

for some small constant $\delta>0$. Then there exist positive constants $T^{\epsilon}\left(0<T^{\epsilon} \leqslant\right.$ $+\infty)$ and $K$, such that the problem (2.7) has a unique classical solution $\left(P^{\epsilon}, \mathbf{U}^{\epsilon}, \Phi^{\epsilon}\right.$, $\mathbf{F}^{\epsilon}, \mathbf{G}^{\epsilon}$ ) satisfying

$$
\begin{gathered}
P^{\epsilon}, \mathbf{U}^{\epsilon}, \Phi^{\epsilon}, \mathbf{F}^{\epsilon}, \mathbf{G}^{\epsilon} \in C^{l}\left(\left[0, T^{\epsilon}\right), H^{s-l}\left(\mathbb{T}^{3}\right)\right), \quad l=0,1 ; \quad \operatorname{div} \mathbf{G}^{\epsilon}=0 \\
\left\|P^{\epsilon}(t)\right\|_{s} \leqslant K \delta, \quad\left\|\Phi^{\epsilon}(t)\right\|_{s} \leqslant K \delta, \quad t \in\left[0, T^{\epsilon}\right) .
\end{gathered}
$$


Notice that for smooth solutions, the non-isentropic Euler-Maxwell system (1.20)(1.24) with initial data (1.26) is equivalent to (2.1) $-(2.6)$ or (2.7) on $[0, T]$. Therefore, in order to obtain the convergence of the Euler-Maxwell system (1.20)-(1.24) to the compressible magnetohydrodynamic equations (1.27)-(1.30), we need to establish the uniform decay estimates in some time interval $[0, T]$ with respect to the parameter $\epsilon$ of the solution to the error system (2.1)-(2.6). We shall present these estimates in the next section.

\section{Uniform ENERGy ESTIMATES AND PROOF OF TheOREM 1.2}

In this section we shall derive the uniform decay estimates with respect to the parameter $\epsilon$ of the solution to the problem (2.1)-(2.6) and justify rigorously the convergence of the non-isentropic Euler-Maxwell system (1.20)-(1.24) to the compressible magnetohydrodynamic equations (1.27)-(1.30). Here we shall make full use of the structure of the system (2.1) - 2.5) and Proposition 2.1. and adapt some techniques developed in [10,12, 13, 20.

We first establish the convergence rate of the error system (2.1)-(2.4) by obtaining the a priori estimates uniformly in $\epsilon$. For simplicity of presentation, we define

$$
\begin{aligned}
\left\|\mathcal{E}^{\epsilon}(t)\right\|_{s}^{2} & =\left\|\left(P^{\epsilon}, \mathbf{U}^{\epsilon}, \Phi^{\epsilon}, \mathbf{G}^{\epsilon}\right)(t)\right\|_{s}^{2}, \\
\left\|\mathcal{E}^{\epsilon}(t)\right\|_{s}^{2} & =\left\|\mathcal{E}^{\epsilon}(t)\right\|_{s}^{2}+\epsilon\left\|\mathbf{F}^{\epsilon}\right\|_{s}^{2}, \\
\left\|\mathcal{E}^{\epsilon}\right\|_{s, T} & =\sup _{0<t<T}\left\|\mathcal{E}^{\epsilon}(t)\right\|_{s} .
\end{aligned}
$$

The crucial estimate of our paper is the following uniform-in- $\epsilon$ result on the error system (2.1)- (2.5).

Proposition 3.1. Let $s>7 / 2$ be an integer and assume that the initial data $\left(P_{0}^{\epsilon}, \mathbf{U}_{0}^{\epsilon}, \Phi_{0}^{\epsilon}, \mathbf{F}_{0}^{\epsilon}, \mathbf{G}_{0}^{\epsilon}\right)$ satisfy

$$
\left\|\left(P_{0}^{\epsilon}, \mathbf{U}_{0}^{\epsilon}, \Phi_{0}^{\epsilon}, \mathbf{G}_{0}^{\epsilon}\right)\right\|_{s}^{2}+\epsilon\left\|\mathbf{F}_{0}^{\epsilon}\right\|_{s}^{2}=\left\|\mathcal{E}^{\epsilon}(t=0)\right\|_{s}^{2} \leqslant M_{0} \epsilon^{2}
$$

for sufficiently small $\epsilon$ and some constant $M_{0}>0$ independent of $\epsilon$. Then, for any $T_{0} \in\left(0, T_{*}\right)$, there are two constants $M_{1}>0$ and $\epsilon_{1}>0$ depending only on $T_{0}$, such that for all $\epsilon \in\left(0, \epsilon_{1}\right]$, it holds that $T^{\epsilon} \geqslant T_{0}$ and the solution $\left(P^{\epsilon}, \mathbf{U}^{\epsilon}, \Phi^{\epsilon}, \mathbf{F}^{\epsilon}, \mathbf{G}^{\epsilon}\right)$ of the problem (2.1)-(2.6), well-defined in $\left[0, T_{0}\right]$, enjoys

$$
\left\|\mathcal{E}^{\epsilon}\right\|_{s, T_{0}} \leqslant M_{1} \epsilon \text {. }
$$

In order to prove Proposition 3.1 we first derive the following a priori estimates on $[0, T]$ with $T \equiv T_{\epsilon}=\min \left\{T_{1}, T^{\epsilon}\right\}$ for some given $\hat{T}<1$ and any $T_{1}<\hat{T}$ independent of $\epsilon$.

\section{1. $L^{2}$ estimates.}

Lemma 3.2. Under the assumptions in Proposition 3.1, it holds that for all $0<$ $t<T$ and sufficiently small $\epsilon$,

$$
\begin{aligned}
& \left\|\left(P^{\epsilon}, \mathbf{U}^{\epsilon}, \Phi^{\epsilon}, \mathbf{G}^{\epsilon}\right)(t)\right\|^{2}+\epsilon\left\|\mathbf{F}^{\epsilon}(t)\right\|^{2}+\frac{3}{2} \int_{0}^{t}\left\|\mathbf{F}^{\epsilon}(\tau)\right\|^{2} \mathrm{~d} \tau \\
\leqslant & C\left\{\left\|\left(P^{\epsilon}, \mathbf{U}^{\epsilon}, \Phi^{\epsilon}, \mathbf{G}^{\epsilon}\right)(t)\right\|^{2}+\epsilon\left\|\mathbf{F}^{\epsilon}(t)\right\|^{2}\right\}(t=0)+C_{T} \epsilon^{2} \\
& +\int_{0}^{t}\left\{\eta_{2}\left\|\mathbf{F}^{\epsilon}(\tau)\right\|^{2}+\eta_{3}\left\|\mathbf{F}^{\epsilon}(\tau)\right\|^{4}+\left[\eta_{1}\left\|\mathbf{F}^{\epsilon}(\tau)\right\|_{2}^{2}+C\left(1+\left\|\mathcal{E}^{\epsilon}(\tau)\right\|_{s}^{2}\right.\right.\right.
\end{aligned}
$$




$$
\left.\left.\left.+\left\|\mathcal{E}^{\epsilon}(\tau)\right\|_{s}^{4}+\left\|\mathcal{E}^{\epsilon}(\tau)\right\|_{s}^{8}\right)\right]\left\|\left(P^{\epsilon}, \mathbf{U}^{\epsilon}, \Phi^{\epsilon}, \mathbf{G}^{\epsilon}\right)(\tau)\right\|^{2}\right\} \mathrm{d} \tau,
$$

where $\eta_{1}, \eta_{2}$, and $\eta_{3}$ are sufficiently small positive constants.

Proof. Multiplying (2.1) by $P^{\epsilon},(2.2)$ by $\mathbf{U}^{\epsilon},(2.3)$ by $\Phi^{\epsilon}$, and integrating them over $\mathbb{T}^{3}$ respectively, we obtain that

$$
\begin{aligned}
& \left\langle a\left(\Phi^{\epsilon}+S^{0}, P^{\epsilon}+p^{0}\right) \partial_{t} P^{\epsilon}, P^{\epsilon}\right\rangle+\left\langle r\left(\Phi^{\epsilon}+S^{0}, P^{\epsilon}+p^{0}\right) \partial_{t} \mathbf{U}^{\epsilon}, \mathbf{U}^{\epsilon}\right\rangle \\
& +\left\langle b\left(\Phi^{\epsilon}+S^{0}, P^{\epsilon}+p^{0}\right) \partial_{t} \Phi^{\epsilon}, \Phi^{\epsilon}\right\rangle \\
= & -\left\langle a\left(\Phi^{\epsilon}+S^{0}, P^{\epsilon}+p^{0}\right)\left(\mathbf{U}^{\epsilon}+\mathbf{u}^{0}\right) \cdot \nabla P^{\epsilon}, P^{\epsilon}\right\rangle \\
& -\left\langle r\left(\Phi^{\epsilon}+S^{0}, P^{\epsilon}+p^{0}\right)\left(\mathbf{U}^{\epsilon}+\mathbf{u}^{0}\right) \cdot \nabla \mathbf{U}^{\epsilon}, \mathbf{U}^{\epsilon}\right\rangle \\
& -\left\langle b\left(\Phi^{\epsilon}+S^{0}, P^{\epsilon}+p^{0}\right)\left(\mathbf{U}^{\epsilon}+\mathbf{u}^{0}\right) \cdot \nabla \Phi^{\epsilon}, \Phi^{\epsilon}\right\rangle \\
& +\left\langle f_{1}^{\epsilon}, P^{\epsilon}\right\rangle+\left\langle\mathbf{f}_{2}^{\epsilon}, \mathbf{U}^{\epsilon}\right\rangle+\left\langle\mathbf{f}_{3}^{\epsilon}, \Phi^{\epsilon}\right\rangle .
\end{aligned}
$$

Thanks to the positivity and smoothness of $a\left(\Phi^{\epsilon}+S^{0}, P^{\epsilon}+p^{0}\right), r\left(\Phi^{\epsilon}+S^{0}, P^{\epsilon}+p^{0}\right)$ and $b\left(\Phi^{\epsilon}+S^{0}, P^{\epsilon}+p^{0}\right)$, Proposition 2.1 the regularity of $\left(p^{0}, \mathbf{u}^{0}, S^{0}, \mathbf{H}^{0}\right)$, CauchySchwarz's inequality, Sobolev's imbedding, and (1.37), we get directly from (2.1), (2.2), and (2.3) that

$$
\begin{aligned}
& \left\|\left(\partial_{t} a\left(\Phi^{\epsilon}+S^{0}, P^{\epsilon}+p^{0}\right), \partial_{t} r\left(\Phi^{\epsilon}+S^{0}, P^{\epsilon}+p^{0}\right), \partial_{t} b\left(\Phi^{\epsilon}+S^{0}, P^{\epsilon}+p^{0}\right)\right)\right\|_{L^{\infty}} \\
\leqslant & \left\|\left(\partial_{t} a\left(\Phi^{\epsilon}+S^{0}, P^{\epsilon}+p^{0}\right), \partial_{t} r\left(\Phi^{\epsilon}+S^{0}, P^{\epsilon}+p^{0}\right), \partial_{t} b\left(\Phi^{\epsilon}+S^{0}, P^{\epsilon}+p^{0}\right)\right)\right\|_{2} \\
\leqslant & \eta_{1}\left\|\mathbf{F}^{\epsilon}\right\|_{2}^{2}+C\left(\left\|\mathcal{E}^{\epsilon}(t)\right\|_{s}^{4}+\left\|\mathcal{E}^{\epsilon}(t)\right\|_{s}^{2}+1\right)
\end{aligned}
$$

for any $\eta_{1}>0$ and

$$
\begin{aligned}
& \left\|\left(\nabla a\left(\Phi^{\epsilon}+S^{0}, P^{\epsilon}+p^{0}\right), \nabla r\left(\Phi^{\epsilon}+S^{0}, P^{\epsilon}+p^{0}\right), \nabla b\left(\Phi^{\epsilon}+S^{0}, P^{\epsilon}+p^{0}\right)\right)\right\|_{L^{\infty}} \\
\leqslant & C\left(1+\left\|\mathcal{E}^{\epsilon}(t)\right\|_{s}+\left\|\mathcal{E}^{\epsilon}(t)\right\|_{s}^{2}\right) .
\end{aligned}
$$

Thus, the first three terms on the right-hand side of (3.4) can be estimated as follows:

$$
\begin{aligned}
& \left|\left\langle a\left(\Phi^{\epsilon}+S^{0}, P^{\epsilon}+p^{0}\right)\left(\mathbf{U}^{\epsilon}+\mathbf{u}^{0}\right) \cdot \nabla P^{\epsilon}, P^{\epsilon}\right\rangle\right| \\
& +\left|\left\langle r\left(\Phi^{\epsilon}+S^{0}, P^{\epsilon}+p^{0}\right)\left(\mathbf{U}^{\epsilon}+\mathbf{u}^{0}\right) \cdot \nabla \mathbf{U}^{\epsilon}, \mathbf{U}^{\epsilon}\right\rangle\right| \\
& +\left|\left\langle b\left(\Phi^{\epsilon}+S^{0}, P^{\epsilon}+p^{0}\right)\left(\mathbf{U}^{\epsilon}+\mathbf{u}^{0}\right) \cdot \nabla \Phi^{\epsilon}, \Phi^{\epsilon}\right\rangle\right| \\
\leqslant & C\left(1+\left\|\mathcal{E}^{\epsilon}(t)\right\|_{s}+\left\|\mathcal{E}^{\epsilon}(t)\right\|_{s}^{2}+\left\|\mathcal{E}^{\epsilon}(t)\right\|_{s}^{4}\right)\left(\left\|P^{\epsilon}\right\|^{2}+\left\|\mathbf{U}^{\epsilon}\right\|^{2}+\left\|\Phi^{\epsilon}\right\|^{2}\right) .
\end{aligned}
$$

By the definition of $f_{1}^{\epsilon}, \mathbf{f}_{2}^{\epsilon}$ and $\mathbf{f}_{3}^{\epsilon}$, the regularity of $\left(p^{0}, \mathbf{u}^{0}, S^{0}, \mathbf{H}^{0}\right)$, and CauchySchwarz's inequality, we have

$$
\begin{aligned}
& \left\langle f_{1}^{\epsilon}, P^{\epsilon}\right\rangle+\left\langle\mathbf{f}_{2}^{\epsilon}, \mathbf{U}^{\epsilon}\right\rangle+\left\langle\mathbf{f}_{3}^{\epsilon}, \Phi^{\epsilon}\right\rangle \\
\leqslant & C \epsilon^{2}+\eta_{2}\left\|\mathbf{F}^{\epsilon}\right\|^{2}+\eta_{3}\left\|\mathbf{F}^{\epsilon}\right\|^{4} \\
& +C\left(1+\left\|\mathcal{E}^{\epsilon}(t)\right\|_{s}^{2}+\left\|\mathcal{E}^{\epsilon}(t)\right\|_{s}^{4}+\left\|\mathcal{E}^{\epsilon}(t)\right\|_{s}^{8}\right)\left(\left\|P^{\epsilon}\right\|^{2}+\left\|\mathbf{U}^{\epsilon}\right\|^{2}+\left\|\Phi^{\epsilon}\right\|^{2}\right)
\end{aligned}
$$

for any $\eta_{2}>0$ and $\eta_{3}>0$.

Putting (3.7) and (3.8) into (3.4) and noticing (3.5), we arrive at

$$
\begin{aligned}
& \left\langle a\left(\Phi^{\epsilon}+S^{0}, P^{\epsilon}+p^{0}\right) P^{\epsilon}, P^{\epsilon}\right\rangle+\left\langle r\left(\Phi^{\epsilon}+S^{0}, P^{\epsilon}+p^{0}\right) \mathbf{U}^{\epsilon}, \mathbf{U}^{\epsilon}\right\rangle \\
& +\left\langle b\left(\Phi^{\epsilon}+S^{0}, P^{\epsilon}+p^{0}\right) \Phi^{\epsilon}, \Phi^{\epsilon}\right\rangle \\
\leqslant & \left\{\left\langle a\left(\Phi^{\epsilon}+S^{0}, P^{\epsilon}+p^{0}\right) P^{\epsilon}, P^{\epsilon}\right\rangle+\left\langle r\left(\Phi^{\epsilon}+S^{0}, P^{\epsilon}+p^{0}\right) \mathbf{U}^{\epsilon}, U^{\epsilon}\right\rangle\right.
\end{aligned}
$$




$$
\begin{aligned}
& \left.+\left\langle b\left(\Phi^{\epsilon}+S^{0}, P^{\epsilon}+p^{0}\right) \Phi^{\epsilon}, \Phi^{\epsilon}\right\rangle\right\}\left.\right|_{t=0}+\int_{0}^{t}\left\{C \epsilon^{2}+\eta_{2}\left\|\mathbf{F}^{\epsilon}\right\|^{2}+\eta_{3}\left\|\mathbf{F}^{\epsilon}\right\|^{4}\right. \\
& \left.+\left[\eta_{1}\left\|\mathbf{F}^{\epsilon}\right\|_{2}^{2}+C\left(1+\left\|\mathcal{E}^{\epsilon}\right\|_{s}^{2}+\left\|\mathcal{E}^{\epsilon}\right\|_{s}^{4}+\left\|\mathcal{E}^{\epsilon}\right\|_{s}^{8}\right)\right]\left\|\left(P^{\epsilon}, \mathbf{U}^{\epsilon}, \Phi^{\epsilon}\right)\right\|^{2}\right\}(\tau) \mathrm{d} \tau .
\end{aligned}
$$

Moreover, we have

$$
\begin{aligned}
& \left\|P^{\epsilon}\right\|^{2}+\left\|\mathbf{U}^{\epsilon}\right\|^{2}+\left\|\Phi^{\epsilon}\right\|^{2} \\
\leqslant & \left\|\left(a\left(\Phi^{\epsilon}+S^{0}, P^{\epsilon}+p^{0}\right)\right)^{-1}\right\|_{L^{\infty}}\left\langle a\left(\Phi^{\epsilon}+S^{0}, P^{\epsilon}+p^{0}\right) P^{\epsilon}, P^{\epsilon}\right\rangle \\
& +\left\|\left(r\left(\Phi^{\epsilon}+S^{0}, P^{\epsilon}+p^{0}\right)\right)^{-1}\right\|_{L^{\infty}}\left\langle r\left(\Phi^{\epsilon}+S^{0}, P^{\epsilon}+p^{0}\right) \mathbf{U}^{\epsilon}, \mathbf{U}^{\epsilon}\right\rangle \\
& +\left\|\left(b\left(\Phi^{\epsilon}+S^{0}, P^{\epsilon}+p^{0}\right)\right)^{-1}\right\|_{L^{\infty}}\left\langle b\left(\Phi^{\epsilon}+S^{0}, P^{\epsilon}+p^{0}\right) \Phi^{\epsilon}, \Phi^{\epsilon}\right\rangle \\
\leqslant & C_{0}\left\{\left\langle a\left(\Phi^{\epsilon}+S^{0}, P^{\epsilon}+p^{0}\right) P^{\epsilon}, P^{\epsilon}\right\rangle+\left\langle r\left(\Phi^{\epsilon}+S^{0}, P^{\epsilon}+p^{0}\right) \mathbf{U}^{\epsilon}, \mathbf{U}^{\epsilon}\right\rangle\right. \\
& \left.+\left\langle b\left(\Phi^{\epsilon}+S^{0}, P^{\epsilon}+p^{0}\right) \Phi^{\epsilon}, \Phi^{\epsilon}\right\rangle\right\},
\end{aligned}
$$

since $a\left(\Phi^{\epsilon}+S^{0}, P^{\epsilon}+p^{0}\right)$ and $r\left(\Phi^{\epsilon}+S^{0}, P^{\epsilon}+p^{0}\right)$ are uniformly bounded away from zero.

Multiplying (2.4) by $\mathbf{F}^{\epsilon}$ and (2.5) by $\mathbf{G}^{\epsilon}$ respectively, and integrating them over $\mathbb{T}^{3}$, we find that

$$
\frac{1}{2} \frac{\mathrm{d}}{\mathrm{d} t}\left(\left\|\sqrt{\epsilon} \mathbf{F}^{\epsilon}\right\|^{2}+\left\|\mathbf{G}^{\epsilon}\right\|^{2}\right)+\int\left(\operatorname{curl} \mathbf{F}^{\epsilon} \cdot \mathbf{G}^{\epsilon}-\operatorname{curl} \mathbf{G}^{\epsilon} \cdot \mathbf{F}^{\epsilon}\right) \mathrm{d} x+\left\|\mathbf{F}^{\epsilon}\right\|^{2}=\left\langle\mathbf{f}_{4}^{\epsilon}, \mathbf{F}^{\epsilon}\right\rangle .
$$

By the regularity of $\left(\mathbf{u}^{0}, \mathbf{H}^{0}\right)$, Cauchy-Schwarz's inequality, and Sobolev's imbedding, the terms on the right-hand side of (3.11) can be bounded by

$$
\frac{1}{4}\left\|\mathbf{F}^{\epsilon}\right\|^{2}+C\left(\left\|\mathcal{E}^{\epsilon}(t)\right\|_{s}^{2}+1\right)\left\|\left(\mathbf{U}^{\epsilon}, \mathbf{G}^{\epsilon}\right)\right\|^{2}+C \epsilon^{2} .
$$

From the fact that

$$
\int\left(\operatorname{curl} \mathbf{F}^{\epsilon} \cdot \mathbf{G}^{\epsilon}-\operatorname{curl} \mathbf{G}^{\epsilon} \cdot \mathbf{F}^{\epsilon}\right) \mathrm{d} x=\int \operatorname{div}\left(\mathbf{F}^{\epsilon} \times \mathbf{G}^{\epsilon}\right) \mathrm{d} x=0,
$$

we get

$$
\begin{aligned}
\frac{1}{2} \frac{d}{d t}\left(\left\|\sqrt{\epsilon} \mathbf{F}^{\epsilon}\right\|^{2}\right. & \left.+\left\|\mathbf{G}^{\epsilon}\right\|^{2}\right)+\frac{3}{4}\left\|\mathbf{F}^{\epsilon}\right\|^{2} \\
& \leqslant C\left(\left\|\mathcal{E}^{\epsilon}(t)\right\|_{s}^{2}+1\right)\left\|\left(\mathbf{U}^{\epsilon}, \mathbf{G}^{\epsilon}\right)\right\|^{2}+C \epsilon^{2} .
\end{aligned}
$$

Note that here we have used the special structure of (2.4) and (2.5).

Thus, we can easily obtain (3.3) by integrating (3.11) and (3.12) over $[0, T]$ and then combining the result with (3.9) and (3.10).

3.2. Higher order estimates on $\Phi^{\epsilon}, \mathbf{F}^{\epsilon}$, and $\mathbf{G}^{\epsilon}$. In order to close the estimate (3.3), we need to derive the higher order estimates of the system (2.1)-(2.5). We first consider the estimates on $\Phi^{\epsilon}, \mathbf{F}^{\epsilon}$, and $\mathbf{G}^{\epsilon}$.

Lemma 3.3. Let the assumptions in Proposition 3.1 hold and the multi-index $\alpha$ satisy $1 \leqslant|\alpha| \leqslant s$. Then, for all $0<t<T$ and sufficiently small $\epsilon$, we have

$$
\begin{aligned}
& \left\|\left(\partial_{x}^{\alpha} \Phi^{\epsilon}, \partial_{x}^{\alpha} \mathbf{G}^{\epsilon}\right)\right\|^{2}+\epsilon\left\|\partial_{x}^{\alpha} \mathbf{F}^{\epsilon}\right\|^{2}+\frac{3}{2} \int_{0}^{t}\left\|\partial_{x}^{\alpha} \mathbf{F}^{\epsilon}(\tau)\right\|^{2} \mathrm{~d} \tau \\
\leqslant & \left\{\left\|\left(\partial_{x}^{\alpha} \Phi^{\epsilon}, \partial_{x}^{\alpha} \mathbf{G}^{\epsilon}\right)\right\|^{2}+\epsilon\left\|\partial_{x}^{\alpha} \mathbf{F}^{\epsilon}\right\|^{2}\right\}(t=0)+C_{T} \epsilon^{2}
\end{aligned}
$$




$$
\begin{aligned}
& +C \int_{0}^{t}\left\{\gamma_{1}\left\|\mathbf{F}^{\epsilon}\right\|_{s}^{4}+\left(\gamma_{2}+\gamma_{3}\right)\left\|\mathbf{F}^{\epsilon}\right\|_{s-1}^{2}+\|\mathcal{E}\|_{s}^{2}+\mathcal{E} \|_{s}^{2 s}\right. \\
& \left.+C\left(1+\|\mathcal{E}\|_{s}^{2(s+1)}\right)\left\|\left(\partial_{x}^{\alpha} \Phi^{\epsilon}, \partial_{x}^{\alpha} P^{\epsilon}, \partial_{x}^{\alpha} \mathbf{U}^{\epsilon}, \partial_{x}^{\alpha} \mathbf{G}^{\epsilon}\right)\right\|^{2}\right\}(\tau) \mathrm{d} \tau
\end{aligned}
$$

where $\gamma_{1}, \gamma_{2}$, and $\gamma_{3}$ are sufficiently small positive constants.

Proof. Dividing (2.3) by $b\left(\Phi^{\epsilon}+S^{0}, P^{\epsilon}+p^{0}\right)$, applying operator $\partial_{x}^{\alpha}(1 \leqslant|\alpha| \leqslant s)$ to the resulting equation, multiplying by $\partial_{x}^{\alpha} \Phi^{\epsilon}$, and integrating over $\mathbb{T}^{3}$, we obtain that

$$
\begin{aligned}
\frac{1}{2} \frac{\mathrm{d}}{\mathrm{d} t}\left\langle\partial_{x}^{\alpha} \Phi^{\epsilon}, \partial_{x}^{\alpha} \Phi^{\epsilon}\right\rangle= & -\left\langle\partial_{x}^{\alpha}\left(\left(\mathbf{U}^{\epsilon}+\mathbf{u}^{0}\right) \cdot \nabla \Phi^{\epsilon}\right), \partial_{x}^{\alpha} \Phi^{\epsilon}\right\rangle \\
& +\left\langle\partial_{x}^{\alpha}\left\{\frac{\mathbf{f}_{3}^{\epsilon}}{b\left(\Phi^{\epsilon}+S^{0}, P^{\epsilon}+p^{0}\right)}\right\}, \partial_{x}^{\alpha} \Phi^{\epsilon}\right\rangle .
\end{aligned}
$$

Now we bound the terms on the right-hand side of (3.14). By the regularity of $\mathbf{u}^{0}$, Cauchy-Schwarz's inequality, and Sobolev's imbedding, we see that

$$
\begin{aligned}
& \left\langle\partial_{x}^{\alpha}\left(\left[\left(\mathbf{U}^{\epsilon}+\mathbf{u}^{0}\right) \cdot \nabla\right] \Phi^{\epsilon}\right), \partial_{x}^{\alpha} \Phi^{\epsilon}\right\rangle \\
= & \left\langle\left[\left(\mathbf{U}^{\epsilon}+\mathbf{u}^{0}\right) \cdot \nabla\right] \partial_{x}^{\alpha} \Phi^{\epsilon}, \partial_{x}^{\alpha} \Phi^{\epsilon}\right\rangle+\left\langle\mathcal{H}^{(1)}, \partial_{x}^{\alpha} \Phi^{\epsilon}\right\rangle \\
= & -\frac{1}{2}\left\langle\operatorname{div}\left(\mathbf{U}^{\epsilon}+\mathbf{u}^{0}\right) \partial_{x}^{\alpha} \Phi^{\epsilon}, \partial_{x}^{\alpha} \Phi^{\epsilon}\right\rangle+\left\langle\mathcal{H}^{(1)}, \partial_{x}^{\alpha} \Phi^{\epsilon}\right\rangle \\
\leqslant & C\left(\left\|\mathcal{E}^{\epsilon}(t)\right\|_{s}+1\right)\left\|\partial_{x}^{\alpha} \Phi^{\epsilon}\right\|^{2}+\left\|\mathcal{H}^{(1)}\right\|^{2},
\end{aligned}
$$

where the commutator

$$
\mathcal{H}^{(1)}:=\partial_{x}^{\alpha}\left(\left[\left(\mathbf{U}^{\epsilon}+\mathbf{u}^{0}\right) \cdot \nabla\right] \Phi^{\epsilon}\right)-\left[\left(\mathbf{U}^{\epsilon}+\mathbf{u}^{0}\right) \cdot \nabla\right] \partial_{x}^{\alpha} \Phi^{\epsilon} .
$$

We use the Moser-type and Cauchy-Schwarz's inequalities, the regularity of $\mathbf{u}^{0}$, and Sobolev's imbedding to infer that

$$
\begin{aligned}
\left\|\mathcal{H}^{(1)}\right\| & \leqslant C\left(\left\|D_{x}^{1}\left(\mathbf{U}^{\epsilon}+\mathbf{u}^{0}\right)\right\|_{L^{\infty}}\left\|D_{x}^{s} \Phi^{\epsilon}\right\|+\left\|D_{x}^{1} \Phi^{\epsilon}\right\|_{L^{\infty}}\left\|D_{x}^{s-1}\left(\mathbf{U}^{\epsilon}+\mathbf{u}^{0}\right)\right\|\right) \\
& \leqslant C\left\|\mathcal{E}^{\epsilon}(t)\right\|_{s}^{2}+C\left\|\mathcal{E}^{\epsilon}(t)\right\|_{s} .
\end{aligned}
$$

By the definiton of $\mathbf{f}_{3}^{\epsilon}$, the last term in (3.14) can be rewritten as

$$
\begin{aligned}
& \left\langle\partial_{x}^{\alpha}\left\{\frac{\mathbf{f}_{3}^{\epsilon}}{b\left(\Phi^{\epsilon}+S^{0}, P^{\epsilon}+p^{0}\right)}\right\}, \partial_{x}^{\alpha} \Phi^{\epsilon}\right\rangle \\
= & -\left\langle\partial_{x}^{\alpha}\left\{\frac{\left[b\left(\Phi^{\epsilon}+S^{0}, P^{\epsilon}+p^{0}\right)-b\left(S^{0}, p^{0}\right)\right]\left[\partial_{t} S^{0}+\mathbf{u}^{0} \cdot \nabla S^{0}\right]}{b\left(\Phi^{\epsilon}+S^{0}, P^{\epsilon}+p^{0}\right)}\right\}, \partial_{x}^{\alpha} \Phi^{\epsilon}\right\rangle \\
& -\left\langle\partial_{x}^{\alpha}\left(\mathbf{U}^{\epsilon} \cdot \nabla S^{0}\right), \partial_{x}^{\alpha} \Phi^{\epsilon}\right\rangle+\left\langle\partial_{x}^{\alpha}\left\{\frac{\left|\mathbf{F}^{\epsilon}+\mathbf{U}^{\epsilon} \times \mathbf{G}^{\epsilon}\right|^{2}}{b\left(\Phi^{\epsilon}+S^{0}, P^{\epsilon}+p^{0}\right)}\right\}, \partial_{x}^{\alpha} \Phi^{\epsilon}\right\rangle \\
& +\left\langle\partial_{x}^{\alpha}\left\{\frac{\left|\mathbf{u}^{0} \times \mathbf{G}^{\epsilon}+\mathbf{U}^{\epsilon} \times \mathbf{H}^{0}\right|^{2}}{b\left(\Phi^{\epsilon}+S^{0}, P^{\epsilon}+p^{0}\right)}\right\}, \partial_{x}^{\alpha} \Phi^{\epsilon}\right\rangle \\
& +\left\langle\partial_{x}^{\alpha}\left\{\frac{2 \mathbf{F}^{\epsilon}}{b\left(\Phi^{\epsilon}+S^{0}, P^{\epsilon}+p^{0}\right)} \cdot\left[\operatorname{curl} \mathbf{H}^{0}+\mathbf{u}^{0} \times \mathbf{G}^{\epsilon}+\mathbf{U}^{\epsilon} \times \mathbf{H}^{0}\right]\right\}, \partial_{x}^{\alpha} \Phi^{\epsilon}\right\rangle \\
& +\left\langle\partial_{x}^{\alpha}\left\{\frac{2\left(\mathbf{U}^{\epsilon} \times \mathbf{G}^{\epsilon}\right)}{b\left(\Phi^{\epsilon}+S^{0}, P^{\epsilon}+p^{0}\right)} \cdot\left[\operatorname{curl} \mathbf{H}^{0}+\mathbf{u}^{0} \times \mathbf{G}^{\epsilon}+\mathbf{U}^{\epsilon} \times \mathbf{H}^{0}\right]\right\}, \partial_{x}^{\alpha} \Phi^{\epsilon}\right\rangle \\
& +\left\langle\partial_{x}^{\alpha}\left\{\frac{2}{b\left(\Phi^{\epsilon}+S^{0}, P^{\epsilon}+p^{0}\right)} \operatorname{curl} \mathbf{H}^{0} \cdot\left(\mathbf{u}^{0} \times \mathbf{G}^{\epsilon}+\mathbf{U}^{\epsilon} \times \mathbf{H}^{0}\right)\right\}, \partial_{x}^{\alpha} \Phi^{\epsilon}\right\rangle
\end{aligned}
$$




$$
:=\sum_{i=1}^{7} \mathcal{I}^{(i)} .
$$

We have to bound the terms on the right-hand side of (3.17). By the regularity of $\left(S^{0}, p^{0}, \mathbf{u}^{0}\right)$, the positivity of $b\left(\Phi^{\epsilon}+S^{0}, P^{\epsilon}+p^{0}\right)$, (1.38), and Cauchy-Schwarz's inequality, the term $\mathcal{I}^{(1)}$ can be bounded as follows

$$
\left|\mathcal{I}^{(1)}\right| \leqslant C\left(\left\|\mathcal{E}^{\epsilon}(t)\right\|_{s}^{2 s}+1\right)\left(\left\|\partial_{x}^{\alpha} \Phi^{\epsilon}\right\|^{2}+\left\|\partial_{x}^{\alpha} P^{\epsilon}\right\|^{2}\right) .
$$

Similarly, the term $\mathcal{I}^{(2)}$ can be controlled by

$$
\left|\mathcal{I}^{(2)}\right| \leqslant C\left(\left\|\mathbf{U}^{\epsilon}(t)\right\|_{s}^{2}+\left\|\partial_{x}^{\alpha} \Phi^{\epsilon}\right\|^{2}\right) .
$$

For the term $\mathcal{I}^{(3)}$, we rewrite it as

$$
\begin{aligned}
\mathcal{I}^{(3)}= & \left\langle\partial_{x}^{\alpha}\left\{\frac{1}{b\left(\Phi^{\epsilon}+S^{0}, P^{\epsilon}+p^{0}\right)}\left|\mathbf{F}^{\epsilon}+\mathbf{U}^{\epsilon} \times \mathbf{G}^{\epsilon}\right|^{2}\right\}, \partial_{x}^{\alpha} \Phi^{\epsilon}\right\rangle \\
= & \left\langle\partial_{x}^{\alpha}\left\{\frac{1}{b\left(\Phi^{\epsilon}+S^{0}, P^{\epsilon}+p^{0}\right)}\left|\mathbf{F}^{\epsilon}\right|^{2}\right\}, \partial_{x}^{\alpha} \Phi^{\epsilon}\right\rangle \\
& +\left\langle\partial_{x}^{\alpha}\left\{\frac{2}{b\left(\Phi^{\epsilon}+S^{0}, P^{\epsilon}+p^{0}\right)} \mathbf{F}^{\epsilon} \cdot\left(\mathbf{U}^{\epsilon} \times \mathbf{G}^{\epsilon}\right)\right\}, \partial_{x}^{\alpha} \Phi^{\epsilon}\right\rangle \\
& +\left\langle\partial_{x}^{\alpha}\left\{\frac{1}{b\left(\Phi^{\epsilon}+S^{0}, P^{\epsilon}+p^{0}\right)}\left|\mathbf{U}^{\epsilon} \times \mathbf{G}^{\epsilon}\right|^{2}\right\}, \partial_{x}^{\alpha} \Phi^{\epsilon}\right\rangle \\
:= & \mathcal{I}^{\left(3_{1}\right)}+\mathcal{I}^{\left(3_{2}\right)}+\mathcal{I}^{\left(3_{3}\right)} .
\end{aligned}
$$

By Cauchy-Schwarz's inequality and Sobolev's embedding, the term $\mathcal{I}^{\left(3_{1}\right)}$ can be bounded by

$$
\begin{aligned}
\mathcal{I}^{\left(3_{1}\right)}= & \left\langle\frac{1}{b\left(\Phi^{\epsilon}+S^{0}, P^{\epsilon}+p^{0}\right)} \partial_{x}^{\alpha}\left(\left|\mathbf{F}^{\epsilon}\right|^{2}\right), \partial_{x}^{\alpha} \Phi^{\epsilon}\right\rangle \\
& +\sum_{\beta \leqslant \alpha,|\beta|<|\alpha|}\left\langle\partial_{x}^{\alpha-\beta}\left(\frac{1}{b\left(\Phi^{\epsilon}+S^{0}, P^{\epsilon}+p^{0}\right)}\right) \partial_{x}^{\beta}\left(\left|\mathbf{F}^{\epsilon}\right|^{2}\right), \partial_{x}^{\alpha} \Phi^{\epsilon}\right\rangle \\
\leqslant & \gamma_{1}\left\|\mathbf{F}^{\epsilon}\right\|_{s}^{4}+C_{\gamma_{1}}\left\|\partial_{x}^{\alpha} \Phi^{\epsilon}\right\|^{2}\left(1+\|\mathcal{E}(t)\|_{s}^{2(s+1)}\right)
\end{aligned}
$$

for any $\gamma_{1}>0$. For the term $\mathcal{I}^{\left(3_{2}\right)}$, by the positivity of $b\left(\Phi^{\epsilon}+S^{0}, P^{\epsilon}+p^{0}\right)$ and Sobolev's imbedding, we have

$$
\begin{aligned}
\mathcal{I}^{\left(3_{2}\right)} & =2\left\langle\partial_{x}^{\alpha} \mathbf{F}^{\epsilon} \cdot \frac{\mathbf{U}^{\epsilon} \times \mathbf{G}^{\epsilon}}{b\left(\Phi^{\epsilon}+S^{0}, P^{\epsilon}+p^{0}\right)}, \partial_{x}^{\alpha} \Phi^{\epsilon}\right\rangle+2\left\langle\mathcal{H}^{(2)}, \partial_{x}^{\alpha} \Phi^{\epsilon}\right\rangle \\
& \leqslant \frac{1}{16}\left\|\partial_{x}^{\alpha} \mathbf{F}^{\epsilon}\right\|^{2}+C\left\|\mathcal{E}^{\epsilon}(t)\right\|_{s}^{2}\left\|\partial_{x}^{\alpha} \mathbf{U}^{\epsilon}\right\|^{2}+2\left\langle\mathcal{H}^{(2)}, \partial_{x}^{\alpha} \Phi^{\epsilon}\right\rangle,
\end{aligned}
$$

where the commutator

$$
\mathcal{H}^{(2)}:=\partial_{x}^{\alpha}\left\{\mathbf{F}^{\epsilon} \cdot \frac{\mathbf{U}^{\epsilon} \times \mathbf{G}^{\epsilon}}{b\left(\Phi^{\epsilon}+S^{0}, P^{\epsilon}+p^{0}\right)}\right\}-\partial_{x}^{\alpha} \mathbf{F}^{\epsilon} \cdot \frac{\mathbf{U}^{\epsilon} \times \mathbf{G}^{\epsilon}}{b\left(\Phi^{\epsilon}+S^{0}, P^{\epsilon}+p^{0}\right)} .
$$

By the Cauchy-Schwarz's and Moser-type inequalities, we obtain that

$$
\begin{aligned}
& 2\left|\left\langle\mathcal{H}^{(2)}, \partial_{x}^{\alpha} \Phi^{\epsilon}\right\rangle\right| \leqslant 2\left\|\mathcal{H}^{(2)}\right\| \cdot\left\|\partial_{x}^{\alpha} \Phi^{\epsilon}\right\| \\
\leqslant & C\left[\left\|D_{x}^{1}\left(\frac{\mathbf{U}^{\epsilon} \times \mathbf{G}^{\epsilon}}{b\left(\Phi^{\epsilon}+S^{0}, P^{\epsilon}+p^{0}\right)}\right)\right\|_{L^{\infty}}\left\|\mathbf{F}^{\epsilon}\right\|_{s-1}\right.
\end{aligned}
$$




$$
\begin{gathered}
\left.+\left\|\mathbf{F}^{\epsilon}\right\|_{L^{\infty}}\left\|\frac{\mathbf{U}^{\epsilon} \times \mathbf{G}^{\epsilon}}{b\left(\Phi^{\epsilon}+S^{0}, P^{\epsilon}+p^{0}\right)}\right\|_{s}\right]\left\|\partial_{x}^{\alpha} \Phi^{\epsilon}\right\| \\
\leqslant \gamma_{2}\left\|\mathbf{F}^{\epsilon}\right\|_{s-1}^{2}+C_{\gamma_{2}}\left(\left\|\mathcal{E}^{\epsilon}(t)\right\|_{s}^{2}+1\right)\left\|\partial_{x}^{\alpha} \Phi^{\epsilon}\right\|^{2} .
\end{gathered}
$$

for any $\gamma_{2}>0$. For the term $\mathcal{I}^{\left(3_{3}\right)}$, by Cauchy-Schwarz's and the Moser-type inequalities, it can be bounded by

$$
\left|\mathcal{I}^{\left(3_{3}\right)}\right| \leqslant C\left(1+\|\mathcal{E}(t)\|_{s}^{2(s+1)}\right)\left\|\left(\partial_{x}^{\alpha} \Phi^{\epsilon}, \partial_{x}^{\alpha} P^{\epsilon}, \partial_{x}^{\alpha} \mathbf{U}^{\epsilon}, \partial_{x}^{\alpha} \mathbf{G}^{\epsilon}\right)\right\|^{2} .
$$

By the regularity of $\left(S^{0}, \mathbf{u}^{0}, \mathbf{H}^{0}\right)$, the positivity of $b\left(\Phi^{\epsilon}+S^{0}, P^{\epsilon}+p^{0}\right)$, and CauchySchwarz's inequality, the terms $\mathcal{I}^{(4)}$ and $\mathcal{I}^{(7)}$ can be bounded as follows:

$$
\left|\mathcal{I}^{(4)}\right|+\left|\mathcal{I}^{(7)}\right| \leqslant C\left(\left\|\mathcal{E}^{\epsilon}(t)\right\|_{s}^{2 s}+1\right)\left\|\left(\partial_{x}^{\alpha} \Phi^{\epsilon}, \partial_{x}^{\alpha} P^{\epsilon}, \partial_{x}^{\alpha} \mathbf{U}^{\epsilon}, \partial_{x}^{\alpha} \mathbf{G}^{\epsilon}\right)\right\|^{2} .
$$

The term $\mathcal{I}^{(5)}$ can be bounded, similarly to $\mathcal{I}^{\left(3_{2}\right)}$, by

$$
\left|\mathcal{I}^{(5)}\right| \leqslant \gamma_{3}\left\|\mathbf{F}^{\epsilon}\right\|_{s-1}^{2}+C_{\gamma_{3}}\left(\left\|\mathcal{E}^{\epsilon}(t)\right\|_{s}^{2}+1\right)\left\|\left(\partial_{x}^{\alpha} \Phi^{\epsilon}, \partial_{x}^{\alpha} P^{\epsilon}, \partial_{x}^{\alpha} \mathbf{U}^{\epsilon}, \partial_{x}^{\alpha} \mathbf{G}^{\epsilon}\right)\right\|^{2}
$$

for any $\gamma_{3}>0$. Finally, similar to $\mathcal{I}^{\left(3_{3}\right)}$, the term $\mathcal{I}^{(6)}$ can be controlled by

$$
\left|\mathcal{I}^{(6)}\right| \leqslant C\left(1+\|\mathcal{E}(t)\|_{s}^{2(s+1)}\right)\left\|\left(\partial_{x}^{\alpha} \Phi^{\epsilon}, \partial_{x}^{\alpha} P^{\epsilon}, \partial_{x}^{\alpha} \mathbf{U}^{\epsilon}, \partial_{x}^{\alpha} \mathbf{G}^{\epsilon}\right)\right\|^{2} .
$$

Substituting (3.15)-(3.26) into (3.14), we conclude that

$$
\begin{aligned}
\frac{1}{2} \frac{\mathrm{d}}{\mathrm{d} t}\left\langle\partial_{x}^{\alpha} \Phi^{\epsilon}, \partial_{x}^{\alpha} \Phi^{\epsilon}\right\rangle \leqslant & C_{\gamma}\left(1+\|\mathcal{E}(t)\|_{s}^{2(s+1)}\right)\left\|\left(\partial_{x}^{\alpha} \Phi^{\epsilon}, \partial_{x}^{\alpha} P^{\epsilon}, \partial_{x}^{\alpha} \mathbf{U}^{\epsilon}, \partial_{x}^{\alpha} \mathbf{G}^{\epsilon}\right)\right\|^{2} \\
& +\gamma_{1}\left\|\mathbf{F}^{\epsilon}\right\|_{s}^{4}+\left(\gamma_{2}+\gamma_{3}\right)\left\|\mathbf{F}^{\epsilon}\right\|_{s-1}^{2}+C\|\mathcal{E}(t)\|_{s}^{2 s}+C\|\mathcal{E}(t)\|_{s}^{2}
\end{aligned}
$$

for some constant $C_{\gamma}>0$ depending on $\gamma_{j}(j=1,2,3)$.

Applying operator $\partial_{x}^{\alpha}(1 \leqslant|\alpha| \leqslant s)$ to (2.4) and (2.5), multiplying the resulting equations by $\partial_{x}^{\alpha} \mathbf{F}^{\epsilon}$ and $\partial_{x}^{\alpha} \mathbf{G}^{\epsilon}$ respectively, and then integrating over $\mathbb{T}^{3}$, we obtain that

$$
\begin{aligned}
& \frac{1}{2} \frac{\mathrm{d}}{\mathrm{d} t}\left(\epsilon\left\|\partial_{x}^{\alpha} \mathbf{F}^{\epsilon}\right\|^{2}+\left\|\partial_{x}^{\alpha} \mathbf{G}^{\epsilon}\right\|^{2}\right)+\left\|\partial_{x}^{\alpha} \mathbf{F}^{\epsilon}\right\|^{2} \\
& +\int\left(\operatorname{curl} \partial_{x}^{\alpha} \mathbf{F}^{\epsilon} \cdot \partial_{x}^{\alpha} \mathbf{G}^{\epsilon}-\operatorname{curl} \partial_{x}^{\alpha} \mathbf{G}^{\epsilon} \cdot \partial_{x}^{\alpha} \mathbf{F}^{\epsilon}\right) \mathrm{d} x \\
= & \left\langle\left[\partial_{x}^{\alpha}\left(\mathbf{U}^{\epsilon} \times \mathbf{H}^{0}\right)+\partial_{x}^{\alpha}\left(\mathbf{u}^{0} \times \mathbf{G}^{\epsilon}\right)\right]-\partial_{x}^{\alpha}\left(\mathbf{U}^{\epsilon} \times \mathbf{G}^{\epsilon}\right), \partial_{x}^{\alpha} \mathbf{F}^{\epsilon}\right\rangle \\
& -\left\langle\epsilon \partial_{x}^{\alpha} \partial_{t} \operatorname{curl} \mathbf{H}^{0}+\epsilon \partial_{x}^{\alpha} \partial_{t}\left(\mathbf{u}^{0} \times \mathbf{H}^{0}\right), \partial_{x}^{\alpha} \mathbf{F}^{\epsilon}\right\rangle .
\end{aligned}
$$

In view of the regularity of $\left(\mathbf{u}^{0}, \mathbf{H}^{0}\right)$, Cauchy-Schwarz's and the Moser-type inequalities, and Sobolev's imbedding, the terms on the right-hand side of (3.28) can be controlled by

$$
\frac{1}{4}\left\|\partial_{x}^{\alpha} \mathbf{F}^{\epsilon}\right\|^{2}+C\left(\left\|\partial_{x}^{\alpha} \mathcal{E}^{\epsilon}(t)\right\|_{s}^{2}+1\right)\left\|\left(\partial_{x}^{\alpha} \mathbf{U}^{\epsilon}, \partial_{x}^{\alpha} \mathbf{G}^{\epsilon}\right)\right\|^{2}+C \epsilon^{2} .
$$

Noticing the fact that

$$
\int\left(\operatorname{curl} \partial_{x}^{\alpha} \mathbf{F}^{\epsilon} \cdot \partial_{x}^{\alpha} \mathbf{G}^{\epsilon}-\operatorname{curl} \partial_{x}^{\alpha} \mathbf{G}^{\epsilon} \cdot \partial_{x}^{\alpha} \mathbf{F}^{\epsilon}\right) \mathrm{d} x=\int \operatorname{div}\left(\partial_{x}^{\alpha} \mathbf{F}^{\epsilon} \times \partial_{x}^{\alpha} \mathbf{G}^{\epsilon}\right) \mathrm{d} x=0,
$$

we find that

$$
\begin{aligned}
& \frac{1}{2} \frac{\mathrm{d}}{\mathrm{d} t}\left(\sqrt{\epsilon} \partial_{x}^{\alpha} \mathbf{F}^{\epsilon}\left\|^{2}+\right\| \partial_{x}^{\alpha} \mathbf{G}^{\epsilon} \|^{2}\right)+\frac{3}{4}\left\|\partial_{x}^{\alpha} \mathbf{F}^{\epsilon}\right\|^{2} \\
& \quad \leqslant C\left(\left\|\mathcal{E}^{\epsilon}(t)\right\|_{s}^{2}+1\right)\left\|\left(\partial_{x}^{\alpha} \mathbf{U}^{\epsilon}, \partial_{x}^{\alpha} \mathbf{G}^{\epsilon}\right)\right\|^{2}+C \epsilon^{2} .
\end{aligned}
$$

We should point out that here we have used the special structure of (2.4) and (2.5).

Combining (3.27) with (3.30), one obtains the estimate (3.13). 
3.3. Higher order estimates on $P^{\epsilon}$ and $\mathbf{U}^{\epsilon}$. In order to close the estimates (3.3) and (3.13), now we study the higher order derivatives of $P^{\epsilon}$ and $\mathbf{U}^{\epsilon}$. We shall adapt the techniques developed in [10,20]. Set

$$
\begin{aligned}
\mathcal{A}\left(\Phi^{\epsilon}, P^{\epsilon}\right) & =\left(\begin{array}{cc}
a\left(\Phi^{\epsilon}+S^{0}, P^{\epsilon}+p^{0}\right) & 0 \\
0 & r\left(\Phi^{\epsilon}+S^{0}, P^{\epsilon}+p^{0}\right) \mathbf{I}_{3}
\end{array}\right) \\
\mathcal{L}\left(\partial_{x}\right) & =\left(\begin{array}{cc}
0 & \operatorname{div} \\
\nabla & 0
\end{array}\right), \quad \mathcal{U}^{\epsilon}=\left(\begin{array}{c}
P^{\epsilon} \\
\mathbf{U}^{\epsilon}
\end{array}\right) .
\end{aligned}
$$

Let $\mathcal{L}_{\mathcal{A}}\left(\partial_{x}\right):=\left\{\mathcal{A}\left(\Phi^{\epsilon}, P^{\epsilon}\right)\right\}^{-1} \mathcal{L}\left(\partial_{x}\right)$. We have

Lemma 3.4. There are constants $K>0$ and $C_{1}>0$ such that for all $\sigma \in\{1, \ldots, s\}$, $\epsilon \in(0,1]$ and $t \in[0, T]$,

$$
\begin{aligned}
& \left\|\mathcal{U}^{\epsilon}\right\|_{\sigma} \leqslant K\left\{\left\|\mathcal{L}\left(\partial_{x}\right) \mathcal{U}^{\epsilon}\right\|_{\sigma-1}+\left\|\operatorname{curl} \mathbf{U}^{\epsilon}\right\|_{\sigma-1}+\left\|\mathcal{U}^{\epsilon}\right\|_{\sigma-1}\right\} \\
& \left\|\mathcal{U}^{\epsilon}\right\|_{\sigma} \leqslant C_{1}\left\{\left\|\left\{\mathcal{L}_{\mathcal{A}}\left(\partial_{x}\right)\right\}^{\sigma} \mathcal{U}^{\epsilon}\right\|_{0}+\left\|\operatorname{curl} \mathbf{U}^{\epsilon}\right\|_{\sigma-1}+\left\|\mathcal{U}^{\epsilon}\right\|_{\sigma-1}\right\} .
\end{aligned}
$$

Proof. (3.31) is obvious. Recalling the fact that $a\left(\Phi^{\epsilon}+S^{0}, P^{\epsilon}+p^{0}\right)$ and $r\left(\Phi^{\epsilon}+\right.$ $\left.S^{0}, P^{\epsilon}+p^{0}\right)$ are smooth, positive, and bounded away from zero with respect to each $\epsilon$, and applying the inequality

$$
\left\|\mathcal{L}\left(\partial_{x}\right) \mathcal{U}^{\epsilon}\right\| \leqslant\|\mathcal{A}\|_{L^{\infty}}\left\|\mathcal{L}_{\mathcal{A}}\left(\partial_{x}\right) \mathcal{U}^{\epsilon}\right\|
$$

we see that (3.32) can be shown easily by induction on $\sigma$.

Next, we bound $\left\|\left\{\mathcal{L}_{\mathcal{A}}\left(\partial_{x}\right)\right\}^{\sigma} \mathcal{U}^{\epsilon}\right\|_{0}$ and $\left\|\operatorname{curl} \mathbf{U}^{\epsilon}\right\|_{\sigma-1}$ by induction. We first show the following estimate.

Lemma 3.5. There exist constants $\bar{s}>0$ and $\kappa_{1}>0$, such that for all $1 \leqslant \sigma \leqslant s$, all $\epsilon \in(0,1]$ and $t \in[0, T]$, it holds that

$$
\begin{gathered}
\int\left(\mathcal{A}\left(\Phi^{\epsilon}, P^{\epsilon}\right)\left|\left\{\mathcal{L}_{\mathcal{A}}\left(\partial_{x}\right)\right\}^{\sigma} \mathcal{U}^{\epsilon}\right|^{2}\right)(t) \mathrm{d} x \leqslant \int\left(\mathcal{A}\left(\Phi^{\epsilon}, P^{\epsilon}\right)\left|\left\{\mathcal{L}_{\mathcal{A}}\left(\partial_{x}\right)\right\}^{\sigma} \mathcal{U}^{\epsilon}\right|^{2}\right)(0) \mathrm{d} x \\
+C \epsilon^{2}+\int_{0}^{t} \kappa_{1}\left\|\mathbf{F}^{\epsilon}(\tau)\right\|_{s}^{4} \mathrm{~d} \tau+C_{\kappa_{1}} \int_{0}^{t}\left(1+\|\mathcal{E}(\tau)\|_{s}^{2 \bar{s}}\right)\left\|\left\{\mathcal{L}_{\mathcal{A}}\left(\partial_{x}\right)\right\}^{\sigma} \mathcal{U}^{\epsilon}(\tau)\right\|^{2} \mathrm{~d} \tau
\end{gathered}
$$

Proof. We follow the arguments in [10,20] with modifications. For simplicity, we set $\mathcal{A}:=\mathcal{A}\left(\Phi^{\epsilon}, P^{\epsilon}\right)$. Let $\mathcal{U}_{\sigma}^{\epsilon}:=\left\{\mathcal{L}_{\mathcal{A}}\left(\partial_{x}\right)\right\}^{\sigma} \mathcal{U}^{\epsilon}, \sigma \in\{1, \ldots, s\}$. It is easy to verify that the operator $\mathcal{L}_{\mathcal{A}}\left(\partial_{x}\right)$ is bounded from $H^{\sigma}$ to $H^{\sigma-1}$ for $\sigma \in\{1, \ldots, s\}$. Note that the equations (2.1) and (2.2) can be written as

$$
\left(\partial_{t}+\left(\mathbf{U}^{\epsilon}+\mathbf{u}^{0}\right) \cdot \nabla\right) \mathcal{U}^{\epsilon}+\frac{1}{\epsilon} \mathcal{A}^{-1} \mathcal{L}\left(\partial_{x}\right) \mathcal{U}^{\epsilon}=\mathcal{A}^{-1} \mathcal{J}^{\epsilon}, \quad \mathcal{J}^{\epsilon}=\left(\begin{array}{c}
f_{1}^{\epsilon} \\
\mathbf{f}_{2}^{\epsilon}
\end{array}\right) .
$$

For $\sigma \geqslant 1$, we commute the operator $\left\{\mathcal{L}_{\mathcal{A}}\right\}^{\sigma}$ with (3.34) and multiply the resulting system by $\mathcal{A}$ to infer that

$$
\mathcal{A}\left(\partial_{t}+\left(\mathbf{U}^{\epsilon}+\mathbf{u}^{0}\right) \cdot \nabla\right) \mathcal{U}_{\sigma}^{\epsilon}+\frac{1}{\epsilon} \mathcal{L}\left(\partial_{x}\right) \mathcal{U}_{\sigma}^{\epsilon}=\mathcal{A}\left(g_{\sigma}^{\epsilon}+\mathbf{h}_{\sigma}^{\epsilon}\right)
$$

where

$$
g_{\sigma}^{\epsilon}:=\left[\partial_{t}+\left(\mathbf{U}^{\epsilon}+\mathbf{u}^{0}\right) \cdot \nabla,\left\{\mathcal{L}_{\mathcal{A}}\right\}^{\sigma}\right] \mathcal{U}^{\epsilon}, \quad \mathbf{h}_{\sigma}^{\epsilon}:=\left\{\mathcal{L}_{\mathcal{A}}\right\}^{\sigma}\left(\mathcal{A}^{-1} \mathcal{J}^{\epsilon}\right) .
$$

Multiplying (3.35) by $\mathcal{U}_{\sigma}^{\epsilon}$ and integrating over $(0, t) \times \mathbb{T}^{3}$ with $t \leqslant T$, and noticing that the singular terms cancel out since $\mathcal{L}\left(\partial_{x}\right)$ is skew-adjoint, we then use the 
inequality (3.5) and Cauchy-Schwarz's inequality to deduce that

$$
\begin{aligned}
\frac{1}{2}\left\langle\mathcal{A}(t) \mathcal{U}_{\sigma}^{\epsilon}(t), \mathcal{U}_{\sigma}^{\epsilon}(t)\right\rangle \leqslant & \frac{1}{2}\left\langle\mathcal{A}(0) \mathcal{U}_{\sigma}^{\epsilon}(0), \mathcal{U}_{\sigma}^{\epsilon}(0)\right\rangle+C \int_{0}^{t}\left(1+\|\mathcal{E}\|_{s}^{4}(\tau)\right)\left\|\mathcal{U}_{\sigma}^{\epsilon}(\tau)\right\|^{2} \mathrm{~d} \tau \\
& +C \epsilon^{2}+\int_{0}^{t}\left(\left\|g_{\sigma}^{\epsilon}(\tau)\right\|_{\sigma}^{2}+\left\|\mathbf{h}_{\sigma}^{\epsilon}(\tau)\right\|_{\sigma}^{2}\right)\left\|\mathcal{U}_{\sigma}^{\epsilon}(\tau)\right\|^{2} \mathrm{~d} \tau
\end{aligned}
$$

Following the proof process of Lemma 2.4 in [20] and applying (3.6), we obtain that

$$
\left\|g_{\sigma}^{\epsilon}(t)\right\| \leqslant C\left(1+\|\mathcal{E}(t)\|_{s}^{2 s_{1}}\right)
$$

for some constant $s_{1}>0$.

Now we estimate the term $\mathbf{h}_{\sigma}^{\epsilon}$. Noticing that there is no spatial derivatives of $\left(P^{\epsilon}, \mathbf{U}^{\epsilon}, \Phi^{\epsilon}, \mathbf{F}^{\epsilon}, \mathbf{G}^{\epsilon}\right)$ in the definitions of $f_{1}^{\epsilon}$ and $\mathbf{f}_{2}^{\epsilon}$, thus we can apply the regularity of $\left(p^{0}, \mathbf{u}^{0}, S^{0}, \mathbf{H}^{0}\right)$, (1.35) and (3.6) to obtain that

$$
\left\|\mathbf{h}_{\sigma}^{\epsilon}(t)\right\| \leqslant \kappa_{1}\left\|\mathbf{F}^{\epsilon}(t)\right\|_{s}^{2}+C_{\kappa_{1}}\left(1+\|\mathcal{E}(t)\|_{s}^{2 s_{1}}\right)
$$

for some constant $s_{2}>0$ and sufficient small $\kappa_{1}>0$.

Putting (3.37) and (3.38) into (3.36) and choosing $\bar{s}=\max \left\{2, s_{1}, s_{2}\right\}$, we get (3.33).

Finally, we derive an estimate for $\left\|\operatorname{curl} \mathbf{U}^{\epsilon}\right\|_{\sigma-1}$. Dividing (2.2) by $r\left(\Phi^{\epsilon}+S^{0}, P^{\epsilon}+\right.$ $\left.p^{0}\right)$ and applying the operator curl to the resulting equations, we obtain that

$$
\begin{aligned}
& {\left[\partial_{t}+\left(\left(\mathbf{U}^{\epsilon}+\mathbf{u}^{0}\right) \cdot \nabla\right)\right]\left(\operatorname{curl} \mathbf{U}^{\epsilon}\right)=\left[\left(\mathbf{U}^{\epsilon}+\mathbf{u}^{0}\right) \cdot \nabla, \operatorname{curl}\right] \mathbf{U}^{\epsilon}} \\
& \quad-\operatorname{curl}\left(\frac{\nabla \Phi^{\epsilon}}{r\left(\Phi^{\epsilon}+S^{0}, P^{\epsilon}+p^{0}\right)}\right)+\operatorname{curl}\left(\frac{\mathbf{f}_{2}^{\epsilon}}{r\left(\Phi^{\epsilon}+S^{0}, P^{\epsilon}+p^{0}\right)}\right) .
\end{aligned}
$$

Lemma 3.6. There exist constants $\overline{\bar{s}}>0$ and $\kappa_{2}>0$, such that the following inequality holds:

$$
\begin{aligned}
\left\|\operatorname{curl} \mathbf{U}^{\epsilon}(t)\right\|_{s-1}^{2} \leqslant & \left\|\operatorname{curl} \mathbf{U}^{\epsilon}(0)\right\|_{s-1}^{2}+C \epsilon^{2}+\int_{0}^{t} \kappa_{2}\left\|\mathbf{F}^{\epsilon}(\tau)\right\|_{s}^{4} \mathrm{~d} \tau \\
& +C_{\kappa_{2}} \int_{0}^{t}\left(1+\|\mathcal{E}(\tau)\|_{s}^{2 \overline{\bar{s}}}\right)\left\|\operatorname{curl} \mathbf{U}^{\epsilon}(\tau)\right\|_{s-1}^{2} \mathrm{~d} \tau
\end{aligned}
$$

Proof. Set $\omega^{\epsilon}:=\operatorname{curl} \mathbf{U}^{\epsilon}$. Taking $\partial_{x}^{\alpha}(0 \leqslant|\alpha| \leqslant s-1)$ to (3.39), multiplying the resulting equations by $\omega^{\epsilon}$, and integrating over $(0, t) \times \mathbb{T}^{3}$ with $t \leqslant T$, we infer that

$$
\begin{aligned}
\frac{1}{2}\left\langle\partial_{x}^{\alpha} \omega^{\epsilon}(t), \partial_{x}^{\alpha} \omega^{\epsilon}(t)\right\rangle \leqslant & \frac{1}{2}\left\langle\partial_{x}^{\alpha} \omega^{\epsilon}(0), \partial_{x}^{\alpha} \omega^{\epsilon}(0)\right\rangle+C \int_{0}^{t}\left(1+\|\mathcal{E}\|_{s}^{4}(\tau)\right)\left\|\partial^{\alpha} \omega^{\epsilon}(\tau)\right\|^{2} \mathrm{~d} \tau \\
& +\int_{0}^{t}\left\langle\left[\left(\mathbf{U}^{\epsilon}+\mathbf{u}^{0}\right) \cdot \nabla, \partial_{x}^{\alpha}\right] \omega^{\epsilon}, \partial_{x}^{\alpha} \omega^{\epsilon}\right\rangle \mathrm{d} \tau \\
& -\int_{0}^{t}\left\langle\partial_{x}^{\alpha} \operatorname{curl}\left(\frac{\nabla \Phi^{\epsilon}}{r\left(\Phi^{\epsilon}+S^{0}, P^{\epsilon}+p^{0}\right)}\right), \partial_{x}^{\alpha} \omega^{\epsilon}\right\rangle \mathrm{d} \tau \\
& +\int_{0}^{t}\left\langle\partial_{x}^{\alpha} \operatorname{curl}\left(\frac{\mathbf{f}_{2}^{\epsilon}}{r\left(\Phi^{\epsilon}+S^{0}, P^{\epsilon}+p^{0}\right)}\right), \partial_{x}^{\alpha} \omega^{\epsilon}\right\rangle \mathrm{d} \tau \\
:= & \frac{1}{2}\left\langle\partial_{x}^{\alpha} \omega^{\epsilon}(0), \partial_{x}^{\alpha} \omega^{\epsilon}(0)\right\rangle+C \int_{0}^{t}\left(1+\|\mathcal{E}(\tau)\|_{s}^{4}\right)\left\|\partial_{x}^{\alpha} \omega^{\epsilon}(\tau)\right\|^{2} \mathrm{~d} \tau \\
& +\int_{0}^{t} \sum_{i=1}^{3} \mathcal{N}_{i}(\tau) \mathrm{d} \tau
\end{aligned}
$$


Next, we estimate the terms $\mathcal{N}_{i}(\tau)(i=1,2,3)$ on the right-hand side of (3.41). From Cauchy-Schwarz's inequality we get that

$$
\left|\mathcal{N}_{1}(\tau)\right| \leqslant C\left\|\partial_{x}^{\alpha} \omega^{\epsilon}(\tau)\right\|\left\|\hat{\mathbf{h}}_{\alpha}^{\epsilon}(\tau)\right\|, \quad \hat{\mathbf{h}}_{\alpha}^{\epsilon}(\tau):=\left[\left(\mathbf{U}^{\epsilon}+\mathbf{u}^{0}\right) \cdot \nabla, \partial_{x}^{\alpha}\right] \omega^{\epsilon} .
$$

The commutator $\hat{\mathbf{h}}_{\alpha}^{\alpha}$ is a sum of terms $\partial_{x}^{\beta}\left(\mathbf{U}^{\epsilon}+\mathbf{u}^{0}\right) \cdot \partial_{x}^{\zeta} \omega^{\epsilon}$ with multi-indices $\beta$ and $\zeta$ satisfying $|\beta|+|\zeta| \leqslant s,|\beta|>0$, and $|\zeta|>0$. Thus,

$$
\left\|\hat{\mathbf{h}}_{\alpha}^{\epsilon}(\tau)\right\| \leqslant C\left(1+\|\mathcal{E}(\tau)\|_{s}^{s}\right),
$$

where the the following nonlinear Sobolev inequality has been used (see [7]): For all $\alpha=\left(\alpha_{1}, \alpha_{2}, \alpha_{3}\right), \sigma \geqslant 0$, and $f, g \in H^{k+\sigma}\left(\mathbb{T}^{3}\right),|\alpha|=k$, it holds that

$$
\left\|\left[f, \partial_{x}^{\alpha}\right] g\right\|_{\sigma} \leqslant C_{0}\left(\|f\|_{W^{1, \infty}}\|g\|_{\sigma+k-1}+\|f\|_{\sigma+k}\|g\|_{L^{\infty}}\right) .
$$

Hence, we have

$$
\left|\mathcal{N}_{1}(\tau)\right| \leqslant C\left(1+\|\mathcal{E}(\tau)\|_{s}^{2 s}\right)\left\|\partial_{x}^{\alpha} \omega^{\epsilon}(\tau)\right\|^{2} .
$$

Noting that basic vector formulas $\operatorname{curl}(\psi \mathbf{u})=\psi \operatorname{curl} \mathbf{u}+\nabla \psi \times \mathbf{u}$ and $\operatorname{curl} \nabla \psi=0$, the term $N_{2}(\tau)$ can be estimated by

$$
\begin{aligned}
\left|\mathcal{N}_{2}(\tau)\right| & \leqslant\left\|\partial_{x}^{\alpha}\left\{\nabla\left(\frac{1}{r\left(\Phi^{\epsilon}+S^{0}, P^{\epsilon}+p^{0}\right)}\right) \times \nabla \Phi^{\epsilon}\right\}\right\|\left\|\partial_{x}^{\alpha} \omega^{\epsilon}(\tau)\right\| \\
& \leqslant C\left(1+\|\mathcal{E}(\tau)\|_{s}^{s_{3}}\right)\left\|\partial_{x}^{\alpha} \omega^{\epsilon}(\tau)\right\|
\end{aligned}
$$

for some $s_{3}>0$, where the properties $r(\cdot, \cdot)$, Proposition 2.1 Sobolev's imbedding, (1.37), and (3.6) have been used.

By the definition of $\mathbf{f}_{2}^{\epsilon}$, the regularity of $\left(p^{0}, \mathbf{u}^{0}, S^{0}, \mathbf{H}^{0}\right)$, Cauchy-Schwarz's inequality, (1.35), (3.6), and (1.37), we have

$$
\left\|\partial_{x}^{\alpha} \operatorname{curl}\left(\frac{\mathbf{f}_{2}^{\epsilon}}{r\left(\Phi^{\epsilon}+S^{0}, P^{\epsilon}+p^{0}\right)}\right)(t)\right\| \leqslant \tilde{\kappa}_{2}\left\|\mathbf{F}^{\epsilon}(t)\right\|_{s}^{2}+C_{\tilde{\kappa}_{2}}\left(1+\|\mathcal{E}(t)\|_{s}^{2 s_{4}}\right)
$$

for some constant $s_{4}>0$ and sufficient small $\tilde{\kappa}_{2}>0$. Thus the term $\mathcal{N}_{3}(\tau)$ can be bounded by

$$
\left|\mathcal{N}_{3}(\tau)\right| \leqslant\left(\tilde{\kappa}_{2}\left\|\mathbf{F}^{\epsilon}(t)\right\|_{s}^{2}+C_{\tilde{\kappa}_{2}}\left(1+\|\mathcal{E}(t)\|_{s}^{s_{4}}\right)\right)\left\|\partial^{\alpha} \omega^{\epsilon}(\tau)\right\| .
$$

Putting (3.41) - (3.44) together, summing up $\alpha$ with $0 \leqslant|\alpha| \leqslant s-1$, applying Cauchy-Schwarz's inequality, and choosing $\overline{\bar{s}}=\max \left\{s_{3}, s_{4}\right\}$ and some sufficient small $\kappa_{2}>0$, we obtain (3.40).

With the estimates in Lemmas 3.23 .6 in hand, we are in a position to prove Proposition 3.1

Proof of Proposition 3.1. As in [12,13,21, we introduce an $\epsilon$-weighted energy functional

$$
\Gamma^{\epsilon}(t)=\left\|\mathcal{E}^{\epsilon}(t)\right\|_{s}^{2} .
$$

Summing up (3.13) with $1 \leqslant|\alpha| \leqslant s$ and (3.33) with $1 \leqslant|\sigma| \leqslant s-1$, combining (3.3) with (3.40), using (3.32) and the fact that $a\left(\Phi^{\epsilon}+S^{0}, P^{\epsilon}+p^{0}\right)$ and $r\left(\Phi^{\epsilon}+S^{0}, P^{\epsilon}+p^{0}\right)$ are smooth, positive, and bounded away from zero with respect to each $\epsilon, \mathbf{F}^{\epsilon} \in$ $C^{l}\left([0, T], H^{s-2 l}\right)(l=0,1)$, and

$$
\left\|\left\{\mathcal{L}_{\mathcal{A}}\left(\partial_{x}\right)\right\}^{\sigma} \mathcal{U}^{\epsilon}\right\|_{0} \leqslant C\left(1+\|\mathcal{E}(\tau)\|_{s}^{s_{5}}\right)\left\|\mathbf{U}^{\epsilon}(\tau)\right\|_{s}
$$


for some $s_{5}>0$, we can choose $\eta_{i}(i=1,2,3), \gamma_{i}(i=1,2,3)$, and $\kappa_{1}, \kappa_{2}$ to be sufficiently small to deduce that there exist a sufficient large constant $s_{0}>0$ and a small $\epsilon_{0}>0$ depending only on $T$, such that for any $\epsilon \in\left(0, \epsilon_{0}\right]$ and $t \in[0, T]$,

$$
\Gamma^{\epsilon}(t) \leqslant C \Gamma^{\epsilon}(t=0)+C \epsilon^{2}+C \int_{0}^{t}\left\{\left(\left(1+\left(\Gamma^{\epsilon}\right)^{s_{0}}\right) \Gamma^{\epsilon}\right\}(\tau) \mathrm{d} \tau .\right.
$$

Thus, applying Gronwall's lemma to (3.45) with the assumption $\Gamma^{\epsilon}(t=0) \leqslant C \epsilon^{2}$ and Proposition 3.1, we obtain that there exist a $0<T_{1}<1$ and an $\epsilon>0$, such that $T^{\epsilon} \geqslant T_{1}$ for all $\epsilon \in(0, \epsilon]$ and $\Gamma^{\epsilon}(t) \leqslant C \epsilon^{2}$ for all $t \in\left[0, T_{1}\right]$. Therefore, the desired a priori estimate (3.2) holds. Moreover, by the standard continuous induction method, we can extend $T^{\epsilon} \geqslant T_{0}$ for any $T_{0}<T_{*}$.

Now we prove Theorem 1.2 by applying Proposition 3.1 .

Proof of Theorem 1.2. By virtue of the definition of the error functions $\left(P^{\epsilon}, \mathbf{U}^{\epsilon}, \Phi^{\epsilon}\right.$, $\left.\mathbf{F}^{\epsilon}, \mathbf{G}^{\epsilon}\right)$, the regularity of $\left(p^{0}, \mathbf{u}^{0}, S^{0}, \mathbf{H}^{0}\right)$, the error system (2.1)-(2.5) and the primitive system (1.20)-(1.24) are equivalent on $[0, T]$ for some $T>0$. Therefore the assumption (1.33) in Theorem 1.2 implies the assumption (3.1) in Proposition 3.1, and hence (3.2) gives (1.34).

Acknowledgements: The authors give their gratitude to professor Hugo Beirão da Veiga for his valuable constructive suggestions on preparation this paper. Jiang was supported by the National Basic Research Program under the Grant 2011CB309705 and NSFC (Grant Nos. 11229101, 11371065). Li was supported by NSFC (Grant No. 11271184), NCET-11-0227, PAPD, and the Fundamental Research Funds for the Central Universities.

\section{REFERENCES}

[1] T. Alazard, Incompressible limit of the nonisentropic Euler equations with the solid wall boundary conditions. Adv. Differential Equations 10 (2005), no. 1, 19-44.

[2] H. Beirão da Veiga, Structural stability and data dependence for fully nonlinear hyperbolic problems, Arch. Ration. Mech. Anal. 120 (1992) 51-60.

[3] H. Beirão da Veiga, Perturbation theorems for linear hyperbolic mixed problems and applications to the Euler compressible equations, Comm. Pure Appl. Math. 46 (1993) 221-259.

[4] H. Beirão da Veiga, A review on some contributions to perturbation theory, singular limits and well-posedness. J. Math. Anal. Appl. 352 (2009), 271-292.

[5] A.C. Eringen, G. A. Maugin, Electrodynamics of Continua II: Fluids and Complex Media, Springer-Verlag, New York, 1990.

[6] W.R. Hu, Cosmic Magnetohydrodynamics, Science Press, Beijing, 1987 (in Chinese).

[7] L. Hömander, Lectures on Nonlinear Hyperbolic Differential Equations, Springer-Verlag, Berlin, 1997.

[8] I. Imai, General principles of magneto-fluid dynamics, in "Magneto-Fulid Dynamics", Suppl. Prog. Theor. Phys, No.24, 1-34, Kyoto Univerity, 1962.

[9] S. Jiang, Q.-C. Ju, F.-C. Li, Low Mach number limit for the multi-dimensional full magnetohydrodynamic equations, Nonlinearity 25 (2012), 1351-1365.

[10] S. Jiang, Q.-C. Ju, F.-C. Li, Incompressible limit of the non-isentropic ideal magnetohydrodynamic equations, available at: arXiv:1301.5126 1 [math.AP].

[11] S. Jiang, Q.-C. Ju and F.-C. Li, Z.-P. Xin Low Mach number limit for the full compressible magnetohydrodynamic equations with general initial data, Adv. Math. 259 (2014), 384-420.

[12] S. Jiang, F.-C. Li, Rigorous derivation of the compressible magnetohydrodynamic equations from the electromagnetic fluid system, Nonlinearity 25 (2012), 1735-1752.

[13] S. Jiang, F.-C. Li, Convergence of the complete electromagnetic fluid system to the full compressible magnetohydrodynamic equations, available at: arXiv:1309.3668 [math.AP] 
[14] S. Kawashima, Smooth global solutions for two-dimensional equations of electromagnetofluid dynamics, Japan J. Appl. Math. 1 (1984), 207-222.

[15] S. Kawashima, Y. Shizuta, Magnetohydrodynamic approximation of the complete equations for an electromagnetic fluid, Tsukuba J. Math. 10 (1986), 131-149.

[16] S. Kawashima, Y. Shizuta, Magnetohydrodynamic approximation of the complete equations for an electromagnetic fluid. II, Proc. Japan Acad. Ser. A Math. Sci. 62 (1986), 181-184.

[17] S. Klainerman, A. Majda, Singular limits of quasilinear hyperbolic systems with large parameters and the incompressible limit of compressible fluids, Comm. Pure Appl. Math. 34 (1981), 481-524.

[18] V. B. Moseenkov, Composition of functions in Sobolev spaces, Ukrainian Math. J. 34 (1982), 316-319.

[19] A. Majda, Compressible Fluid Flow and Systems of Conservation Laws in Several Space Variables. Springer-Verlag, New York, 1984.

[20] G. Métivier, S. Schochet, The incompressible limit of the non-isentropic Euler equations, Arch. Ration. Mech. Anal. 158 (2001), 61-90.

[21] Y.J. Peng, S. Wang, Rigorous derivation of incompressible e-MHD equations from compressible Euler-Maxwell equations, SIAM J. Math. Anal. 40 (2008), 540-565.

[22] B. Rubino, Singular limits in the data space for the equations of magneto-fluid dynamics. Hokkaido Math. J. 24 (1995), no. 2, 357-386.

[23] P. Secchi, On the singular incompressible limit of inviscid compressible fluids. J. Math. Fluid Mech. 2 (2000), 107-125.

[24] P. Secchi, On slightly compressible ideal flow in the half-plane. Arch. Ration. Mech. Anal. 161 (2002), no. 3, 231-255.

[25] S. Schochet, The compressible Euler equations in a bounded domain: existence of solutions and the incompressible limit. Comm. Math. Phys. 104 (1986), no. 1, 49-75.

[26] A. I. Vol'pert, S. I. Hudjaev, On the Cauchy problem for composite systems of nonlinear differential equations, Math. USSR-Sb. 16 (1972) 517-544.

Institute of Applied Physics and Computational Mathematics, P.O. Box 8009, Beijing 100088, P.R. CHINA

E-mail address: jiang@iapcm.ac.cn

Department of Mathematics, Nanjing University, Nanjing 210093, P.R. China

E-mail address: fli@nju.edu.cn 